(c) <2021 > . This manuscript version is made available under the CC-BY-NC-ND 4.0 license http://creativecommons.org/licenses/by-nc-nd/4.0/

The definitive publisher version is available online at https://doi.org/ 10.1016/j.istruc.2021.08.004 


\section{Graphical Abstract}

Integrated computational framework for the design and fabrication of bending-active structures made from flat sheet material

Francesco Laccone,Luigi Malomo,Nico Pietroni,Paolo Cignoni,Tim Schork

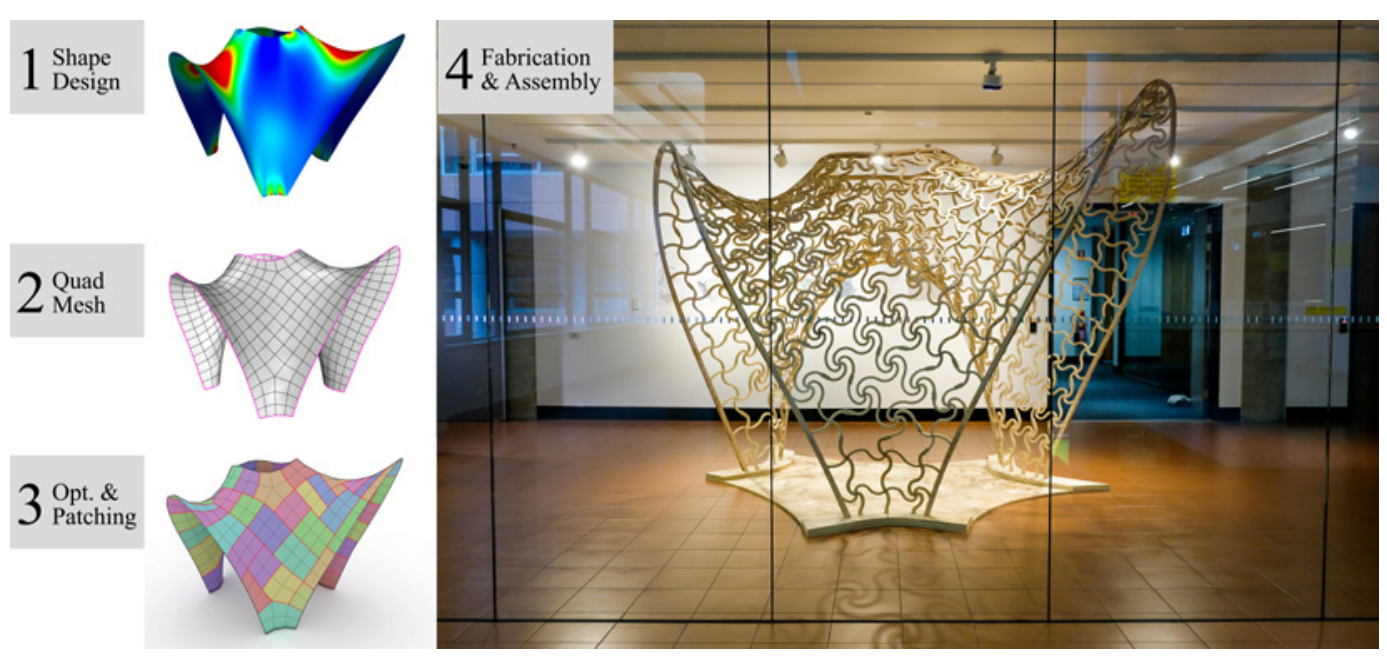


Highlights

Integrated computational framework for the design and fabrication of bending-active structures made from flat sheet material

Francesco Laccone,Luigi Malomo,Nico Pietroni,Paolo Cignoni,Tim Schork

- Novel architectural method for the automatic design and optimization of bending-active structures from an arbitrarilycurved input shape.

- The FlexMaps algorithm is modified and extended at the architectural scale to provide optimized spiral mesostructures.

- Automated shape-to-factory workflow for advanced manufacturing.

- Application in a 1:1 scale proof of concept demonstrator. 


\title{
Integrated computational framework for the design and fabrication of bending-active structures made from flat sheet material
}

\author{
Francesco Laccone ${ }^{a, b, *}$, Luigi Malomo ${ }^{a}$, Nico Pietroni $^{c}$, Paolo Cignoni $^{a}$ and $\operatorname{Tim}_{\text {Schork }}{ }^{d}$ \\ ${ }^{a}$ ISTI, CNR, via G. Moruzzi 1, 56124 Pisa, Italy \\ ${ }^{b}$ DESTEC, University of Pisa, Largo Lazzarino 2, 56122 Pisa, Italy \\ ${ }^{c}$ School of Computer Science, Faculty of Engineering and Information Technology (FEIT), University of Technology Sydney (UTS), 15 Broadway Ultimo, \\ Sydney Australia \\ ${ }^{d}$ Transformative Technologies Lab, School of Architecture, Faculty of Design, Architecture and Building (DAB), University of Technology Sydney (UTS), \\ 15 Broadway Ultimo, Sydney Australia
}

\section{ARTICLE INFO}

\section{Keywords:}

computational design

simulation

advanced manufacturing

structural design

digital fabrication

shell

optimization

form-finding

mesostructured

architecture

\begin{abstract}
A B S T R A C T
This paper introduces an integrated computational design framework for the design and realization of arbitrarily-curved bending-active architectural structures. The developed framework consists of a series of methods that enable the production of a complex 3D structures composed of a set of flat 2D panels whose mechanical properties are locally tuned by varying the shape of embedded spiraling patterns. The resulting panels perform as variable stiffness elements, and they are optimized to match a desired target shape once assembled together. The presented framework includes all the steps for the physical delivery of architectural objects, including conception, static assessment, and digital fabrication. The developed framework has been applied to an architectural scale prototype, which demonstrates the potential of integrating architectural design, computational simulation, structural engineering, and digital fabrication, opening up several possible novel applications in the building sector.
\end{abstract}

\section{Introduction}

It is estimated that, due to worldwide population growth and urbanization, approximately 2.6 billion people will require new housing by 2050 . This demand will be impossible to meet due to the required natural resources and the current low and stagnating productivity of the construction industry. Furthermore, the built environment is responsible for nearly $40 \%$ of global greenhouse gas emission [2,3]. Our future challenge is finding solutions that allow to build more with less material, using renewable resources that create less greenhouse gases while at the same time creating high-quality architectural solutions. These solutions include efficient structural solutions that take advantage of the material properties. The fields of Architectural Geometry and advanced geometry processing offer new approaches to solving some of these challenges. Architectural Geometry is a new interdisciplinary topic that has gained a significant boost in recent years $[4,5]$. This research field manages problems related to the design, optimization, and fabrication of complex architectural shapes. The complexity originates from the different constraints that shape aesthetics, structural design, and engineering aspects of a desired structure pose. Thus, the paneling of free-form shapes [6, 7], the assembly of self-supporting and interlocking structures $[8,9,10]$ or the structural design of shells and grid shells $[11,12,13,14,15,16,17]$ have become non-trivial prob-

\footnotetext{
*Corresponding author

Q francesco.laccone@isti.cnr.it;

francesco. laccone@destec. unipi.it (F. Laccone)

ORCID(s): $0000-0002-3787-7215$ (F. Laccone); $0000-0001-7892-894 \mathrm{X}$

(L. Malomo); 0000-0002-8271-2102 (N. Pietroni); 0000-0002-2686-8567 (P.

Cignoni); 0000-0002-4583-2439 (T. Schork)
}

lems. A new generation of computational design workflows takes advantage of geometry processing methods to support the exploration of the space of solutions that conform to mechanics, fabrication, or budget limitations.

The quests for compelling free-form shapes in architecture drove architectural geometry to focus on practical issues, such as material, production, fabrication and assembly [18]. Hence, one of the relevant problems is that usually the individual components are considerably smaller than the final structure. Therefore, the subdivision and discretization of the continuous target shape represents a key step towards the building feasibility and deserves a large research effort. A common strategy is to investigate the piecewise approximation of complex surfaces or their developability [19, 20,21].

An alternative approach is the use of mesostructures to fulfill specific shape requirements through mechanical deformation. Such structures work at an intermediate scale (hence the term meso-), which is not the material scale (micro-), and can be appropriately designed to reproduce the mechanical response of a different material by simply using varying geometry. For instance, material can be selectively removed from a solid plate or a volume to create elements with a custom deformation capacity. Such a product is also referred as meta-material, since a target behavior is obtainable in spite of the original base-material property. This induced non-homogeneity provides the opportunity to achieve a large number of variations by altering the initial geometry, which in turn means that specialized meta-materials can be adopted locally to comply with specific required performances. This is one of the base strategies of the functionally graded material concept in design [22].

While the meta-material approach has been successfully used 
for the production of small scale objects [23, 24], its full potential in the architectural context is still unexplored. A key advantage of the use of mesostructures in architecture is that they enable common building materials to achieve a greater deformation capacity, and so they expand the space of fabricable designs. On the other hand, mesostructures require particular attention on the related structural issues, since they have to achieve a certain deformed shape and at the same time they should bear external loads. In the architectural context, similar issues are usually addressed in the bending-active structures.

We adopted the algorithm [1], which is tailored to the digital fabrication of small-scale objects, and we extended it to work at architectural scale. Shifting the focus to a larger scale poses several exciting new challenges in terms of material requirements, structural behavior, fabrication design, and assembly. We can recap the main contribution of this paper as follows.

- we proposed a novel architectural pipeline for the automatic design, optimization and fabrication of bendingactive structures from smooth, even doubly-curved, input shapes;

- we extended the FlexMaps method [1] to work at the architectural scale;

- our pipeline includes an important step based on nonlinear Finite Element (FE) analysis to assess the produced internal stress due to form-giving bending and due to the design load;

- the proposed pipeline consists in a shape-to-factory workflow, which eventually generates the path for subtractive manufacturing machines;

- we tested and applied the developed pipeline in a 1:1 scale proof of concept demonstrator.

\subsection{Bending-active structures}

Bending-active structures are a promising solution to efficiently achieve doubly-curved architectural surfaces out of flat sheets or straight elements. In bending-active structures, the elements are directed to a global self-equilibrating status by the elastic deformations caused by joint bending and stretching. The curvature and the internal stress produced in the equilibrium configuration are key drivers of the entire design process. In general, given a base material, the formdefining process evaluates each elements' shape to match a certain equilibrium configuration, as well as a user-provided set of boundary constraints. Additionally, the employed material needs high-breaking strain capacity [25]. This scenario makes bending-active structures not trivial to design. Modern bending-active structures were pioneered mainly by adopting geometry-based approaches, in which the material status is evaluated analytically based on the estimated element curvature. Examples of this structures are the Mannheim Multihalle by Frei Otto built in 1974, the largest post-formed wooden grid shell; and the Plydome Structures introduced by Buckminster Fuller in 1957, which are geodesic spheres made from single-curved plywood plates.

Applications of these structures have been limited for a long time due to the lack of computational tools and methods capable of simulating their complex structural behavior. The increase of computing power and further sophistication of digital fabrication technology have led to a recent renaissance and renewed interest in bending-active structures [26, 27]. Bending-active structures are a cost-effective construction technique to produce geometrically complex and highly efficient lightweight structures [28, 29, 30, 31]. The backbone of the digital design workflow for bending-active structure is a new generation of geometry- and material- driven methods $[32,33,34,35,36]$. Until recently the majority of physically-based methods offer limited or no shape control to the designer. Newly developed methods make it possible to set a target shape. These methods search for the best setting or configuration of the load-bearing elements such that, once manufactured and deployed, they form a structure that closely approximate the target shape.

The work by La Magna [37] exploits the bending stiffness of plates and their deformation modes. His first contribution (form-conversion approach) provides a method for covering the target shape with bent plates by manipulating the topology, allowing simple developable elements to map multiple directions. This is a general method that guarantees the bendability of the elements and their ability to cover the whole surface space. The second contribution (embedded approach) exploits the deformation properties of cellular structures to design meta-materials that can replicate the base surface's curvature.

The recent X-Shells proposed by Panetta et al. [38] enables the computational design of deployable structures made of continuous, elastically bent beams that are connected by rotational joints and assembled in a flat grid configuration. A specific actuation system forces the beams to twist and buckle out-of-plane to reach a desired target position at the static equilibrium. The assembled flat state and the deployed configuration are jointly optimized, while a sparse distribution of actuation forces is subsequently optimized to ease the erection phase.

FlexMaps [1] is a fully automated method to optimize the stiffness of a particular class of mesostructures. The method discretizes the surface into flat, spiral-shaped, deformable sub-modules. Once these are actively bent, the final structure in equilibrium state results as close as possible to the target shape.

\subsection{Architectural challenges and objectives}

Originally the FlexMaps method [1] was developed for the design and fabrication of small-scale objects. A further study attempted to use use this approach in a larger scale prototype project $[39,40]$.

The research presented in this paper builds on this work and further extends it by exploring and testing the developed approach to a more complex topology. The main aim of the project is to consolidate the use of FlexMaps in advanced 


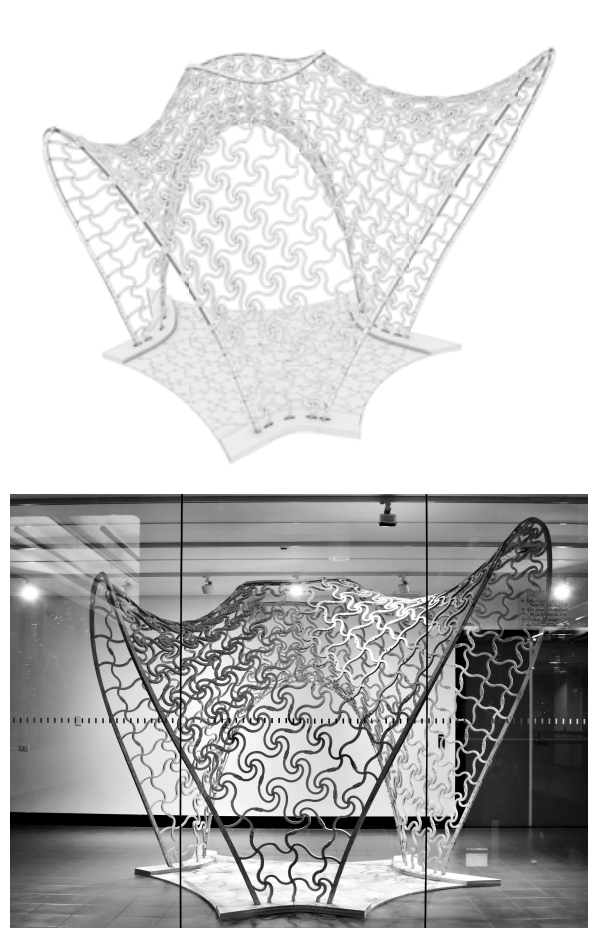

Figure 1: Computer rendering (top) and view of the fully assembled FlexMaps 2.0 demonstrator (bottom).

architectural design and further integrate and streamline the workflow that links shape design to digital fabrication. The integrated computational design framework extends the previous method and includes shape design, optimization, structural verification, 2D layout design, and digital manufacturing. The developed framework was tested in a demonstrator project that was built at the School of Architecture, Faculty of Design, Architecture, and Building (DAB) of the University of Technology Sydney (Fig. 1). The whole structure is made out of CNC-milled $15 \mathrm{~mm}$ thick plywood components.

\section{FlexMaps algorithm}

FlexMaps optimizes the shape of individual patterns to compose 2D mesostructured panels, which deform in a prescribed manner. Each pattern is shaped as a four-arms spiral which can be embedded in a quadrilateral mesh face (i.e. four spiraling arms connect the face barycenter to the midpoints of the quad edges). Such patterns can stretch and bend in any direction and also assume double curvature. The approach starts by quadrangulating the input shape. To reduce the number of required pieces, a semi-automatic merging process connects adjacent quads, which are then clustered and mapped from the input surface onto a planar domain. A spiral-shaped mesostructure is embedded into each flat quad. A reduced physical data-driven model is used to estimate the mechanical behavior of each individual spiraling pattern, with respect to the parameters determining its shape. This model enables the simulation of the resulting shape in $3 \mathrm{D}$ at interactive rates, as well as the automatic optimization of the patterns' parameters for improving the quality of the shape approximation.

Using a four-arms spiral pattern as mesostructure has three main advantages:

- As opposed to simple straight elements, spirals offer less resistance to bending, making them well-suited to approximate high curvatures, as they distribute internal stress in a controlled way.

- The mechanical behavior of a spiral can be locally tuned by modifying just two geometrical parameters, the spiral angle and wall thickness (see Section 3.3). Consequently, keeping the constituting material fixed, a mesostructured panel's stiffness can be tuned on a local basis by acting on the geometry of each spiral.

- The spiral connectivity results very simple and makes it possible to connect one element with their neighbors even in case of mesh singularities (Fig. 2).

Eventually we obtain a set of panels containing a limited number of spirals, namely patches, and each one is optimized to have the entirely-assembled structure match the target shape. Essentially, the algorithm produces a set of anisotropic patches that, assembled together, are capable of reaching the desired shape by uniformly redistributing their internal stress. This stress is the one induced during the assembly phase, by individually bending and mutually connecting these patches.
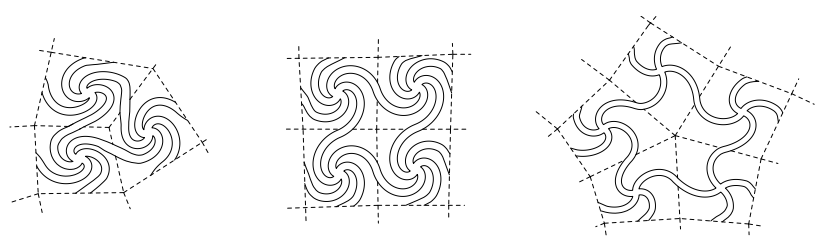

Figure 2: Connections between the spirals in the case of 3 quads (singularity), 4 quads (regular case), 5 quads (singularity).

\section{Design method}

Besides the design capabilities of the FlexMaps method, the architectural scenario analyzed in this paper poses additional challenges ranging from the shape conception to the final physical validation at the architectural scale. The main steps of the new workflow for the architectural scenario is presented in Fig. 3 and it consists of five steps:

shape design In this initial step the architect designs a freeform continuous target shape. While modeling, the designer should consider several constraints involved, such as the maximum size, the overall weight and the anchor points of the resulting structure. Moreover, being a lattice structure the shape should be at least stable.

quadrilateral remeshing As in FlexMaps, the present pipeline requires an initial remeshing step that is performed 


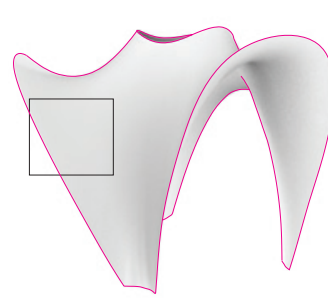

(a)

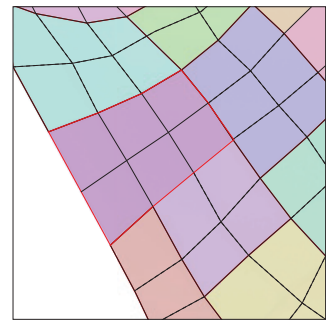

(b)

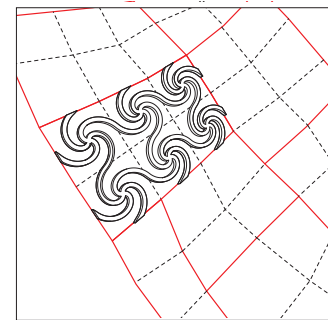

(c)

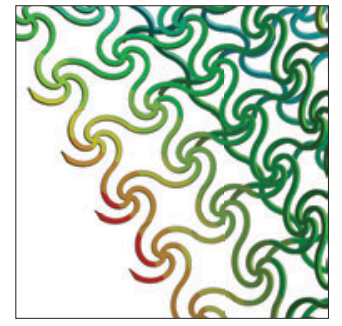

(d)

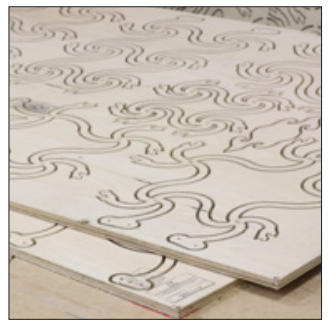

(e)

Figure 3: Our workflow: (a) shape design, (b) quadrilateral remeshing, (c) pattern shape optimization, (d) structural analysis, (e) fabrication and assembly.

using a technique similar to [41]. The remeshing is guided by an underlying curvature field and optimizes for having quads as regular as possibile. This is a crucial aspect for the correct characterization of the embedded patterns, as the reduced simulation model is computed from spirals embedded into regular quads and results inaccurate for deformed shapes.

spiral form-finding This step optimizes the shape of each individual pattern in order to match the expected target geometry. As in FlexMaps this step requires two main components: a way to characterize how the shape of a spiral affects its physical behavior, and a method to derive the desired elastic properties such that the final assembled structure matches the target shape. Those two problems are strictly interconnected. As in FlexMaps the behavior of a spiral pattern is parametrized with respect to its geometric parameters, it is simulated and it is represented using a reduced model for fast forward simulation, while backward derivation is used for the global shape optimization. This component is similar to the original FlexMaps pipeline [1] but it has been redesigned to account for a different scale, novel building materials, additional design load (e.g., gravity) that can be considered at this stage, and a more user-friendly way to specify the scenario's boundary conditions.

structural analysis A structural analysis is used to validate the static performance of the structure, namely once submitted to the form-giving bending and self weight. This FE analysis uses a non-linear orthotropic material model to simulate the behaviour of the plywood and a well-tested, reliable pipeline to perform it for multiple components and large displacements.

fabrication and assembly The final step includes the generation of 2D milling instructions for manufacturing and the actual assembling of the entire structure.

\subsection{Shape design}

The architect is free to design complex target shapes to be approximated and fabricated via the proposed pipeline. In theory there are no restrictions on the topology, Gaussian curvature, supports and loading conditions of the target design. However, in practice, the achievable accuracy for the resulting shape approximation is affected by several factors:

- The resolution of the mesostructure influences the capacity of the structure to approximate high frequency geometries, such as sharp edges.

- The designer should consider that the material can eventually break if it undergoes an excessive load or an excessive target deformation.

- The pipeline will not produce a close approximation of the input design if its shape and boundary conditions (e.g., loading scenario plus anchor points) are far from being physically achievable.

Targeting the architectural scale includes also two additional specific challenges:

- From a geometric point of view, architectural-scale objects, being surfaces with boundaries, are more difficult to produce because preserving the 'open-boundaries' requires additional devices (i.e., edge beams) or fixing anchor points. In contrast, for small-scale objects such problems are usually neglected.

- Similarly to the point above, the weight of small-scale structures is negligible, and stability is not the most relevant issue. In contrast, these are fundamental requirements for architectural-scale objects. Indeed, it is not possible to reuse a simulation setup targeted to small-scale structures [34], nor is it possible to rescale or adjust the cross-sections of the patterns at a later stage for a bigger scale, as this has a direct impact on the bendability and material strength.

Consequently, the optimization has to be performed on the object's real scale and the actual permanent load, which is neglected in [1], has to be considered. However, since the optimization process does not explicitly manage the input shape's stability, an additional design constraint is to consider the initial shape definition to be at least stable in the equivalent continuum. 
Integrated computational framework for bending-active structures

\subsection{Quadrilateral remeshing and patch segmentation}

The problem of transforming 2D patches into assembled $3 \mathrm{D}$ structures is related to developability, namely the property of 3D mesh to be unrolled and flattened without distortion on a 2D plane, either entirely or subdivided into patches. This 3D-to-2D mapping is referred in computer graphics as parametrization [42], and usually the accompanying distortion is a quantity to minimize. Our parametrization procedure starts by tessellating the desired surface geometry (e.g., NURBS) into a triangulated mesh. Then, it generates quad mesh strips by tracing curvature-aligned geodesic paths directly onto the triangle mesh, using the method in [41], producing a quadrangulation as a byproduct. As explained in Section 3, FlexMaps assumes that mesostructures are embedded in regularly-shaped quads. Consequently, the more regular are the quads, the more accurate is the reduced model simulation. For this reason, the method described in [12] is applied as a post-processing regularization of the quadrangulation.

After a shape is converted into a regularized quad mesh, a semi-automatic process groups adjacent quads, producing a set of quad patches which are then mapped with low distortion onto a flat domain. In this way it is possible to decompose even non-developable complex shapes into a set of flat patches which will become the building blocks of the target structure. If desired, the user can manually tune this patch decomposition with a simple visual interface.

\subsection{Spiral patterns optimization}

The scope of this step is to tune the parameters of the mesostructures such that the globally assembled shape approximates as closely as possible the designed target shape.

The FlexMaps algorithm (refer to [1] for the details) relies on three local geometric parameters for each spiral pattern (Fig. 4 top): the pattern size $(s)$, which is implicitly set in the quadrangulation phase; the spiral twist angle $(\theta)$; and the spiral wall thickness $(w)$. By varying the spiral parameters within a fixed quad size, a matrix of spiral morphologies with differentiated material behavior can be created (Fig. 4 bottom). For example, a combination of low wall thickness and a high spiral twist angle results in a highly flexible spiral, required, for example, in areas with high curvature. In contrast, large spiral wall thickness and low spiral angle result in a stiff spiral, which makes sense for flat regions. Simulations are performed for a large set of patterns and different load scenarios and the resulting precomputed data is used to populate a parametric database with a sampling of the achievable spiral shapes and their relative mechanical behavior.

The optimization algorithm determines the heterogeneous distribution of spirals by interpolating patterns from this precomputed database. A data-driven two-scale model is used to predict and then optimize, in a continuous space, the approximate physical behavior of our structures. At the fine level, the algorithm parametrically generates an accurate geometric model of a single spiral structure and simulate its behavior using a non-linear FE model with thousands of de-
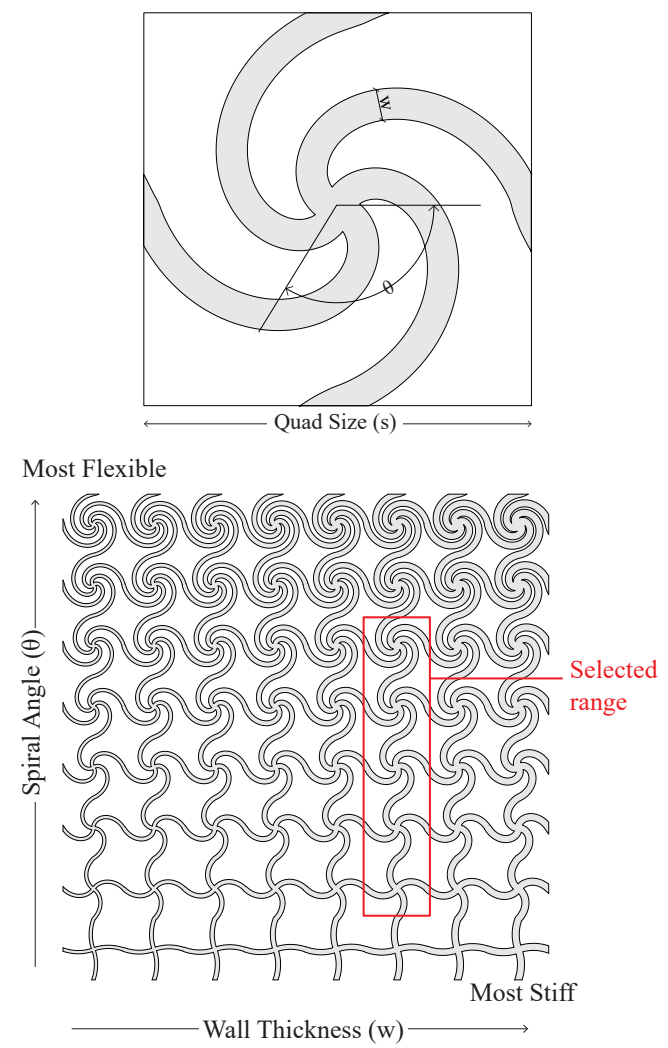

Figure 4: Spiral parameters (top) and matrix of spiral morphologies (bottom).

grees of freedom. At the coarse level, such simulation data is used to produce a simple beam model that captures axial, bending and twisting deformation modes and can be efficiently integrated into a sensitivity analysis for global shape optimization. The data resulting from this phase consists of the full set of patches containing optimized spiral mesostructures, laid out as flat surfaces.

\subsection{Structural analysis}

The optimization routine gives no control over punctual stress and provides only limited control over the resulting deformation. With an acceptable accuracy loss, the procedure employs an efficiently reduced model that allows the overall computation to be tractable. However, at the architectural scale these considerations raise the need for a detailed structural analysis. This active shell is modeled employing solid finite elements to obtain its exact deformation, to verify that the stress is within the capacity of the employed material, and to estimate the overall safety level. This verification workflow has been built for the ANSYS software environment [43] and consists of two simulation phases, which are conceptually depicted in Fig. 5.

In the first phase (Fig. 5a), every flat patch is rigidly moved from the horizontal plane, in which it is generated, to a position that is as close as possible to its corresponding deformed target shape. This patch positioning improves the convergence of the analysis since a limited number of time 


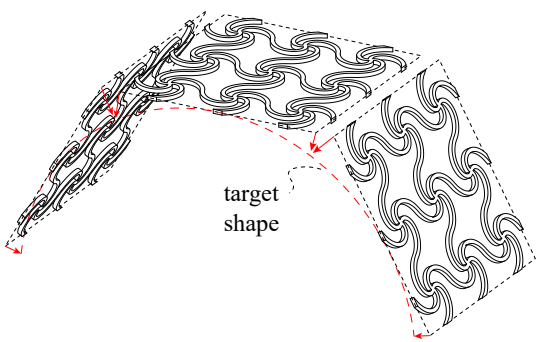

(a)

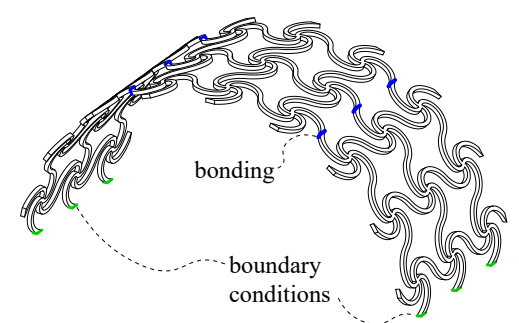

(b)

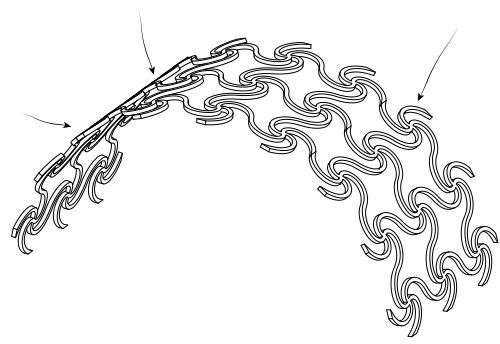

(c)

Figure 5: Strategy adopted for the structural analysis on an exemple structure of an arch made of three all-equal mesostructured patches: (a) the flat panels are moved as close as possible to the target shape, then each of them is deformed; (b) the patches are bonded and boundary conditions are imposed at the extremities; (c) external loading can be applied.

steps are needed to complete the nonlinear simulation. This phase approximates the form-giving bending behavior that each individual spiral patch will be subjected to during assembly. A displacement is imposed at the extremities of the spirals' free arms to move them into their target deformed shape. Consequently, all spirals will independently assume their pre-stressed actively-bent configuration (as in Fig. 5b). The purpose of this initial procedure is not to simulate the exact assembly sequence but to provide a solution that approximates the strain of each spiral during the formation process (all patches are simultaneously bent independently). Modeling the assembly sequence is a much harder problem. It requires considering the actual assembly sequence, the collisions, and all the deformations imposed to bring the panels to their final position.

After this first step, the resulting states of the panels (deformed shapes and accompanying strains) are set as starting point of the second phase, in which it is simulated the behavior of the structure as a whole. The individually-bent patches are linked, and boundary conditions are applied (Fig. 5b). The patches are then released so they can uniformly redistribute their accumulated stress, the gravity, and other loads applied under, such as its self-weight or in general all loads that are assumed as permanent (Fig. 5c).

For performing such simulations it is recommended to deduce the material properties to plug into the model from experimental tests on real-scale patches prototypes (Fig. 5 shows an example of such prototypes for calibrating the material properties). This is particularly critical as subtractive manufacturing processes may lead to a reduced and more scattered stiffness in the case of composite or natural materials. Moreover, the stiffness and strength of the connectors must be evaluated experimentally via bending and tension/compression tests with the objective of conceive a detail that restores the cross-section characteristics.

\subsection{Fabrication and construction system}

FlexMaps enables both additive and subtractive manufacturing. Additive manufacturing is undoubtedly a material saving strategy, but it is not recommended at the architectural scale since the local material strength and continuity are fun- damental. Defects should be avoided as they can affect the shape and or propagate in failures. On the other hand, subtractive manufacturing is better suited as it can rely on a large number of materials to be actively bent and produced in form of flat sheets, i.e., aluminum, plywood, glass; but a drawback is that the gaps between the spirals became material waste. Good quality can be obtained with a reduced manufacturing time using $\mathrm{CNC}$ machines, such as laser and water-jet cutters or milling machines, and integrating the digital fabrication into the current pipeline.

The manufactured structure reaches its final shape under appropriate boundary conditions once all patches, carved from flat sheets, are individually bent and tied together. In particular, the connection between the patches should be able to guarantee full-strength and preserve this capacity during the time, i.e. not going into creep. An illustration of the construction system is shown in Fig. 6.

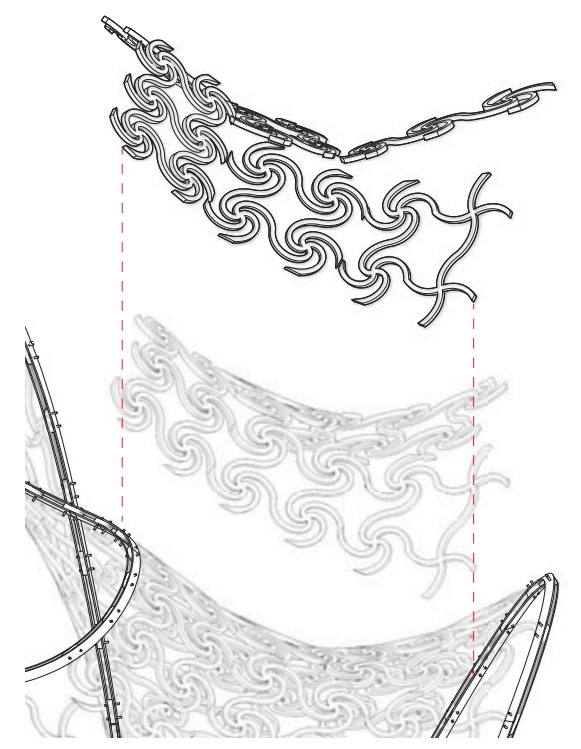

Figure 6: Conceptual exploded view of the FlexMaps bendingactive system and its assembly: the flat mesostrucrured panels are bent and tied together; this mutual constraint and appropriate boundary conditions hold them in the desired position within the structure. 


\section{Architecture of the computational model}

To connect the operations of the presented methodology, a centralized computational model has been developed as shown in Fig. 7. All aspects of the design and development process are integrated into it. This model is made of a core part and several subroutines. Moreover, to initiate the design process, some preliminary actions are required, i.e. the stability check of the equivalent-continuum target shape and its quad remeshing. General considerations are also important in this phase to inform the computational model.

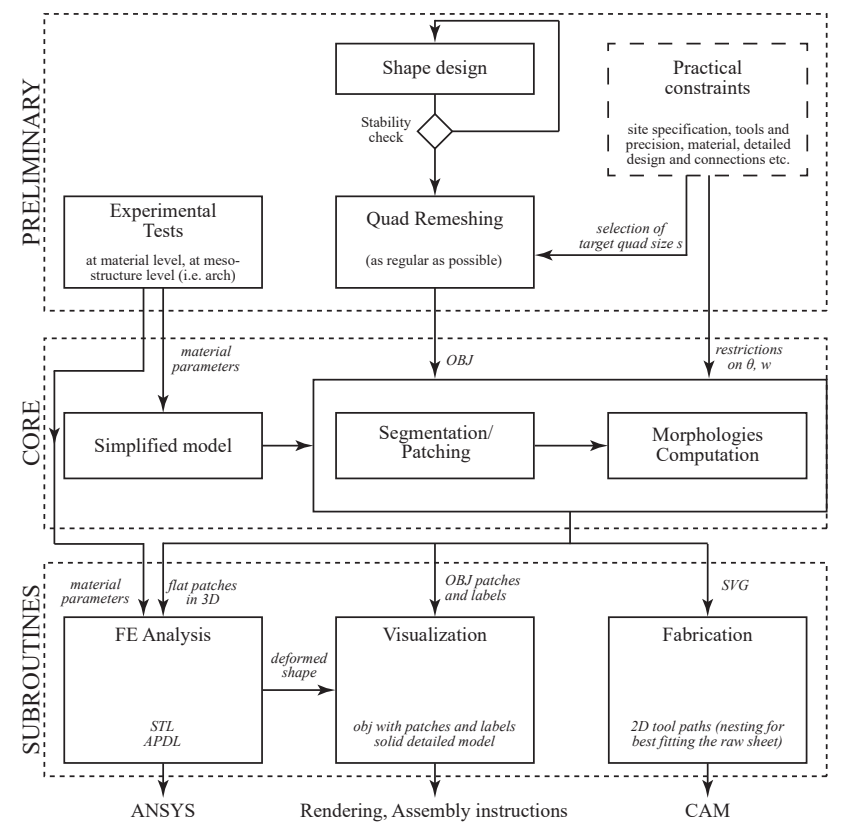

Figure 7: Schematic representation of the flow chart in the computational model.

The core part is a script and include the steps developed in the work FlexMaps [1]. It consists of the implementation of the reduced model, the shape optimization routines, and the system for producing the flat quad patches.

The FE Analysis subroutine is aimed at generating input for the ANSYS software, namely a geometry file and a list of instructions in the form of APDL commands. For instance, this latter contains deformation vectors to bring the points of the connectors from the flat shape to their candidate deformed shape (first phase of the analysis, Section 3.4). For generating the geometry of the panels, all parametrized 2D mesh patches are moved to a position close to their ideal deformed shape. Then, a Grasshopper [44] script has been developed to convert discrete meshes into NURBS and to generate solid geometries, which can be subsequently processed in the FE analysis.

The output of the visualization subroutine is a geometrical model to be used for navigation and for rendering. Also a mesh model with distinct annotated patches is automatically built and it is aimed to guide the assembly of the structure. The raw result of the core process is an array of solid meshes. This data is inherently compatible with additive fabrication processes, in which the provided mesh can be easily 3D printed. On the other hand, subtractive fabrication processes need a dedicated subroutine since the essential part is not the solid piece but its outline (indeed, the outlines identify tool paths). In this subroutine, at first the meshes' outlines are converted into NURBS for a better interoperability with most CAM softwares, then the connectors are generated at the endpoints of patch-boundary spirals (the core process ignores the presence of connectors). Additionally, cutting tolerances can be embedded, as well as the specification of using different machine tools for different cuts (i.e. different diameters tools can be selected based on the desired precision and stored as different layers).

The computational model has allowed an easy exploration of the demonstrator feasibility and has been of fundamental help in supporting decisions and coordinating all the design and fabrication choices required for such a complex project. The model shows also wide flexibility for what the geometric design space is concerned. Conversely, the material input is restricted to a specific database, which needs to be recomputed each time a new material is considered.

\section{The Demonstrator}

The method feasibility was tested and evaluated at the architectural scale by building a full scale prototype (Fig. 1). As project constraints, this demonstrator could not rely on hanging supports from the ceiling or any rigid fixing. The system has been conceived to be in equilibrium by just standing on the floor. Gravity load is considered as acting in both the form-giving process and as the only load during the lifespan of the demonstrator. It consists of:

- a set of mesostructured flat panels that are optimized through the algorithm;

- a base made of three supporting edge beams and an interlocking floor system to tie these beams together;

- a set of slender edge beams to preserve the relative position of connectors located on the surface's open boundary.

For these purposes we selected plywood as building material, as it represented a balancing point between ease of manufacturing, cost and mechanical requirements. Therefore, all components of the demonstrator have been CNC-milled out of $15 \mathrm{~mm}$ thick plywood sheets, and this process has been carried out entirely within UTS faculty facilities. The manufactured panels resulted lightweight and easy to handle even for unskilled workers and did not require any special handling equipment. Among common building materials, plywood is listed as suitable to be used in active-bent contexts due to a good proportion of flexural strength and stiffness $[25,45]$.

\subsection{Experimental tests}

The fist step required to initiate the design process is to perform experimental tests to characterize the building material and calibrate the base material properties. A good test 
case is a three-panels arch, made of $3 \times 9$ all-equal spirals and similar to the example of Fig. 5. This simple structure has been manufactured and tested in two setups with same boundary and loading conditions, but with two different spans of $1.5 \mathrm{~m}$ and $2.0 \mathrm{~m}$ (Fig. 8 (a) and (b) respectively). The supports are cylindrical hinges, so they allow rotations and avoid vertical and horizontal displacement. For each setup, the three mid-span nodes are loaded in three steps of $0.5,1.0$ and $1.5 \mathrm{~kg}$ per node. For each load increment the deformations are measured through a Artec handheld scanner. The load is materialized as steel blocks hanged from the center of each of the three mid-span spirals (as visible in Fig. 8). The objective is to have two structures with different stiffness to be tested at different loading rates, having fabricated only three patches. The stiffness of the spirals depends on various factors related to their geometry and to the material locally. For this specimen the spirals are designed to be representative of the mean properties of the demonstrator, so they are inscribed in $0.25 \times 0.25 \mathrm{~m}$ quads, and have fixed parameters $\theta=125^{\circ}$ and $w=15 \mathrm{~mm}$. The specimen in the assembled and developed configuration measures $0.75 \times 2.25 \mathrm{~m}$. The material properties have been obtained by reverse fitting the measured deformation on a FE model as per Fig. 5 (developed in two phases, following the same strategy presented for the full model in Section 3.4), given as target points the mid-span nodes deformations in the three load steps. The material properties are obtained by multiplying reference values by a factor until simulated and measured displacements result as close as possible.

A first FE model uses an isotropic material, which is a simplification needed to calibrate the reduced model adopted in the core process. This is a requirement for guaranteeing a consistent computation of the optimized spirals morphologies. Besides, a second FE model uses a more realistic orthotropic material, whose parameters are intended to be used in the full simulation of the demonstrator. Hence, a higher accuracy is needed to assess the internal stress and predict the actual deformed shape.

In any case, these experiments can be regarded as a test bench of the whole pipeline and are fundamental to familiarize with the constructional system, to verify the detailed design and in general to gather essential knowledge to face all practical issues that may arise while working on larger structures. Since the selected target size of quads and all the details, such as the connectors, are the same of the demonstrator, this tests set the benchmark for the tools, the speed and precision of machine milling as well.

\subsection{Shape design and optimization}

For the design of the structure a set of NURBS-based shapes have been considered as candidates, in particular, to challenge the physical production of a complex topology within a limited indoor space (Fig. 9). The shapes are either manually modeled or obtained through form-finding techniques. Eventually we selected the red-framed geometry in Fig. 9 because it has a significant variation of curvature, despite its small size, and has no symmetries. The selected anticlastic design is a non-developable vaulted surface with three outer arch profiles and a central hole. The arches have different heights and are linked to a central hole using saddle-shaped surfaces that merge reciprocally. The Gaussian curvature of the surface flips as it approaches the ground on three different supporting lines (Fig. 10a). The whole shape fits a volume of $3.41 \times 3.02 \times 2.71$ meters, which was compatible with the available exposition space.

The initial quadrangulation and patch decomposition has been performed according to the method described in Section 3.2. At first, a smooth cross-field has been computed following the main curvature directions and aligned with the borders (Fig. 10b). Then, a mainly regular quadrangulation has been derived (Fig. 10c), except for few singular vertices, where a valence of 3 or 5 is necessary to comply with positive or negative Gaussian curvature. The patch layout has been generated using the semi-automatic process that iteratively merges quads together into flattened patches by keeping the introduced distortion below a given threshold. In the demonstrator, for aesthetic and practical reasons, some patch boundaries have been manually enforced using the dedicated graphical tool (Fig. 11a).

Due to fabrication constraints, we have selected a target quad edge of $s=0.26 \mathrm{~m}$. This led to a spiral size that is manageable during fabrication, and also easy to handle and bend during the assembly. Moreover, for aesthetic reasons and to avoid local weakening, the optimization space of spiral parameters has been restricted to a constant width of $w=$ $15 \mathrm{~mm}$ and a twist range of $\theta=40-180^{\circ}$ (red frame in Fig. 4). Eventually, each spiral arm can have a different length but a constant cross-section of $15 \times 15 \mathrm{~mm}$ to guarantee a minimum stiffness everywhere. The upper limit on the twist angle serves instead to avoid excessively-dense spirals, whose arms may result too close to the milling tolerance. The angle lower bound, instead, avoids aesthetically unpleasant spirals, resulting similar to a cross.

\subsection{Detailed design}

The connector between the patches is a fundamental detail of the system. This connection is required to be fullstrength and therefore substain axial, shear, bending and torsion. At the same time, the connection mechanism has to be easy to assemble and aesthetically pleasant. The adopted key connection (Fig. 12a bottom) is made of two antisymmetric interlocking parts. The contact surface friction and the shape interlocking provide a sufficient resistance in preparation for the ultimate lock of the pieces by means of a couple of through-hole M5 bolts (Fig. 12a top). The bolts work as a key that avoids any relative movement. The drilling precision and fabrication tolerance are extensively calibrated as they have a major impact on the connection stiffness and, therefore, on the success of the assembly.

The open boundaries of the structure have been stiffened with edge beams. Each edge reinforcement is made of a couple of plywood lamellae, which are point-fixed on the end-point of the spiral free arms located at the boundaries (Fig. 12b). Here, the spiral arms are equipped with two 


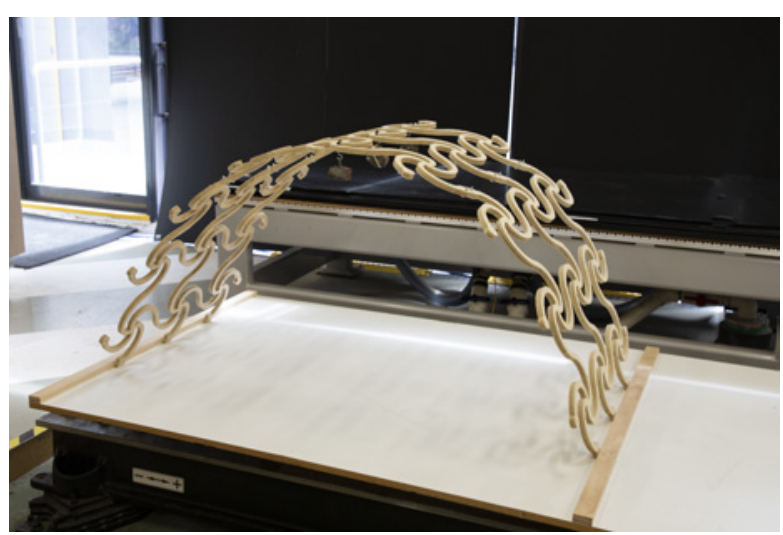

(a)

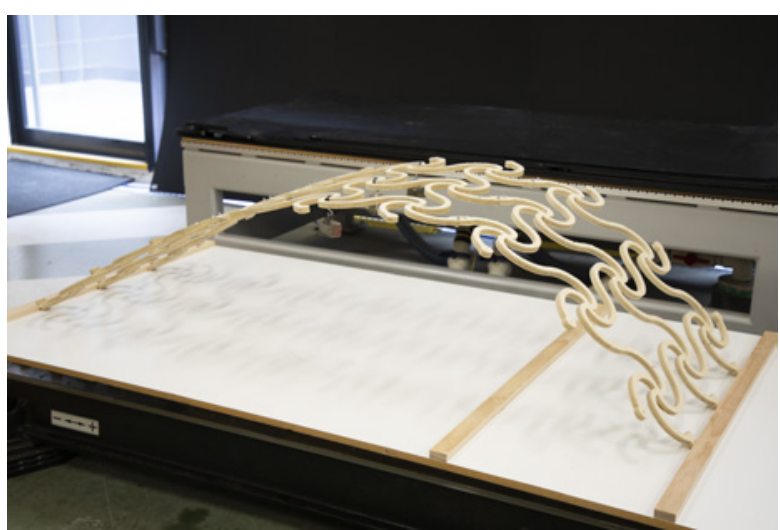

(b)

Figure 8: Experimental test on a three-panels arch made of all equal spirals, the structure is loaded on the three spirals of the mid section with $0.5,1.0$ and $1.5 \mathrm{~kg}$ : (a) first setup with a span of $1.5 \mathrm{~m}$; (b) second setup with a span of $2.0 \mathrm{~m}$.

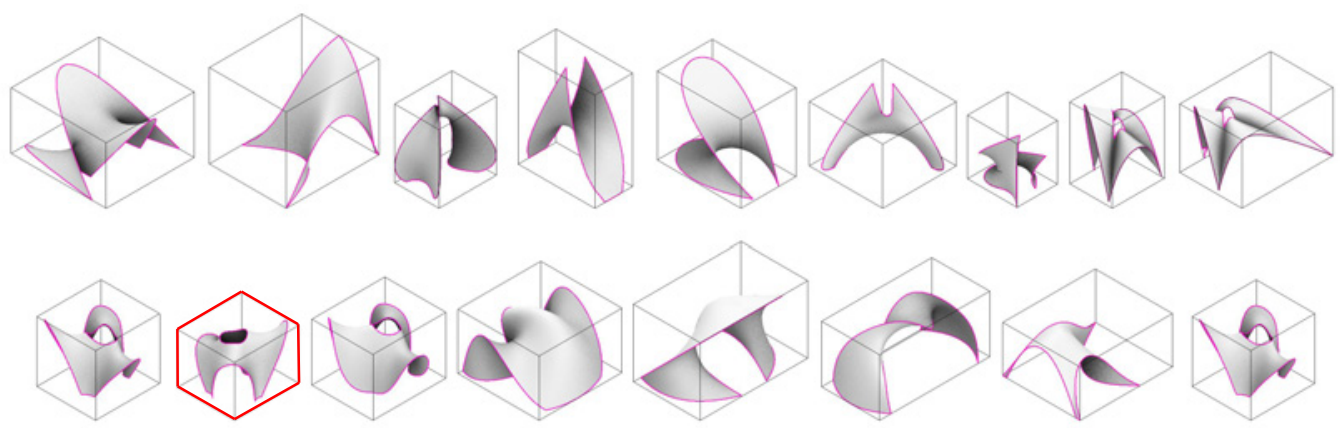

Figure 9: Shape alternatives explored for the design of the demonstrator (the selected shape is framed in red).

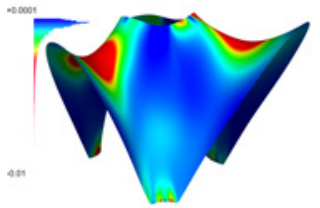

(a)

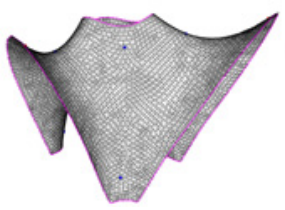

(b)

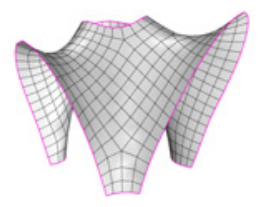

(c)
Figure 10: Quadrangulation process: (a) gaussian curvature; (b) curvature-aligned cross-field; (c) quadrangulation with a selected resolution.

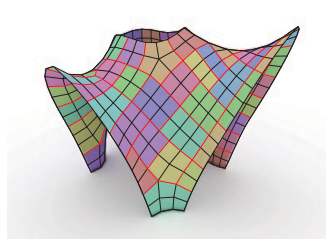

(a)

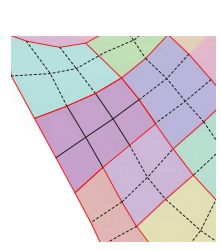

(b)
Figure 11: Quads clustered in patches (a) and closeup on one patch with its mesostructured actively-bent panel (b).

holes for the bolts that hold the two lamellae. The seams of the two lamellae are interleaved between the two sides of the whole boundary path, to preserve a smooth profile and avoid kinks. The primary function of these edge beams is to preserve a correct inter-distance between the boundary connectors. Additionally, they help the patches to preserve their target deformed shape during the assembly. The profiles are geometrically modeled based on the target deformed shape, i.e. the result of the phase one of the structural analysis. For manufacturing, a $6 \mathrm{~mm}$ plywood panel has been used, so each edge lamella cross-section is $6 \times 30 \mathrm{~mm}$. It is worth noting that edge beams are also actively bent but, since their bending-stiffening contribution to spiral patches has been observed to be low, their contribution has not been considered in the FE structure simulation.

Concerning the floor support structure, the base spirals terminate with $\mathrm{T}$-shaped extremities that are embedded into ground beams made of three $15 \mathrm{~mm}$ plywood plies (Fig. 12c). The first two plies provide the T-extremities with the right shape tangency, while the top ply avoids their uplift. Each central ply of the ground beam hides two ballast iron discs that have been designed to provide a sufficient bending supportreaction. Once all these components are dry assembled, the base plies are screwed together.

All bottom layers of the ground beams have an interlocking design that allows them to connect to the floor. The latter 
is a wooden tiling milled from the same $15 \mathrm{~mm}$ panels employed for the spirals and the ground beams. The design of the floor is based on the aperiodic Penrose pattern shown in Fig. 13. The rhombus tiles are modified with an interlocking joint to hold the edge beams of the bases and counteract the thrust forces introduced by the structure. The connection mechanism is dry and demountable. The interlocking tiles have redundant connectivity because they are designed to provide a diffuse restraint to the thrust forces induced by the base spirals.

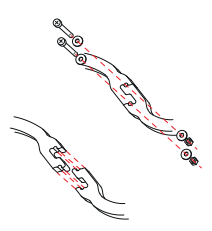

(a)

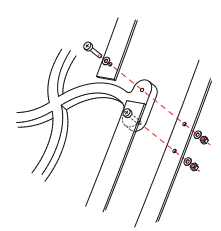

(b)

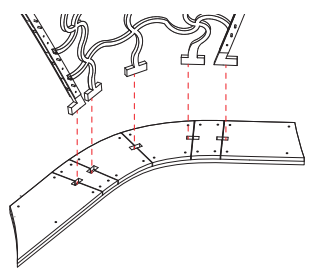

(c)
Figure 12: Detailed design: (a) connector between patches; (b) edge beam connector and lamellae; (c) panel footings.

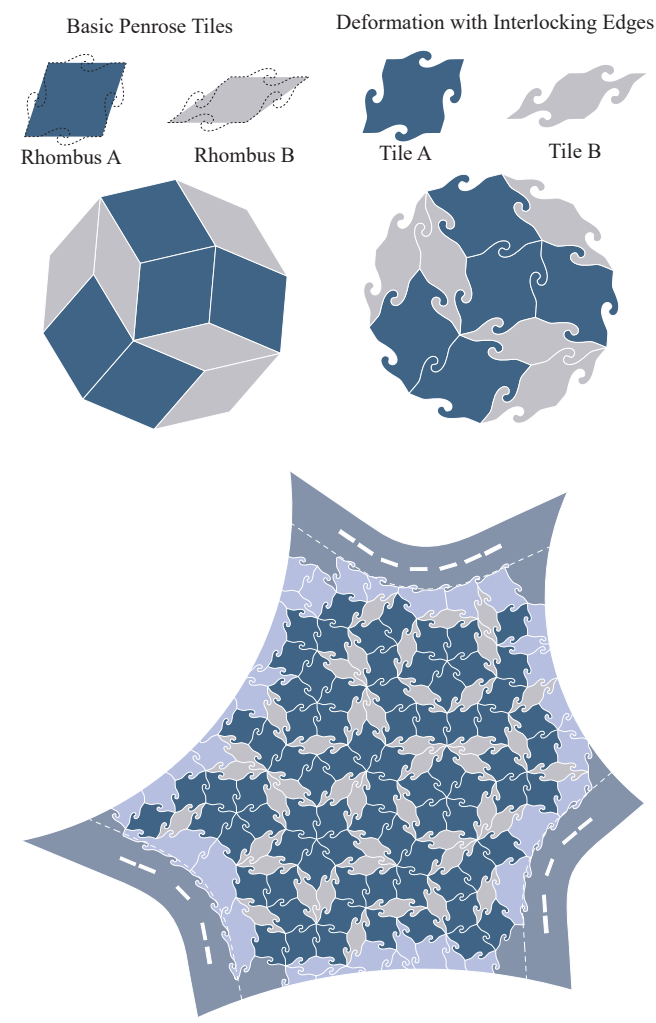

Figure 13: Rhomboid Penrose aperiodic pattern generation (top) and paving design (bottom).

\subsection{Modeling and structural analysis results}

With the exception of edge beams, all the demonstrators' wooden components have been machined from $15 \mathrm{~mm}$ plywood, composed of hardwood and softwood plies. The material properties adopted in the analyses are derived from the experimental tests performed on the arch specimen (Section 5.1), from which orthotropic material properties are derived (Table 1).

The demonstrator patches are generated as 2D meshes, which are then moved to their starting positions and orientation (as shown in Section 3.4), from which their solid geometry is generated. The latter is imported in the ANSYS software package and is re-meshed by means of finite brick elements. Each solid patch is equipped with a local reference system to correctly orient the material anisotropy. This information has been extracted from the fabrication subroutine, in which the patches are nested and oriented on the raw panels to be processed by the milling machine. It is worth mentioning that the patches are not located on the milling plane to pursue a mechanical criterion but rather a cost- and timesaving one. As for the manufacturing, the spiral optimization does not consider the material anisotropy and therefore the material strength and the shape accuracy have to be carefully checked via FE analysis.

The analysis results are shown in Fig. 14. In phase one, the patches are bent through the imposed displacement of up to $115 \mathrm{~mm}$. As predicted, this phase produces stress peaks in the connection points, which are expected to mutually balance once spiral arms are connected inter-patch. Phase two simulates the actual behavior of the assembled demonstrator, inheriting deformation and strains from the previous phase. Some modeling simplifications are introduced, especially for the connections details. The patches connector (Fig. 12a) is reduced to a face-to-face coupling, in which a bonding constraint applies to simulate a full-strength connection. The connection of the spiral arm to the edge beam (Fig. 12b) is replaced by springs that link pairs of neighboring arms. This modeling is conservative since only the axial stiffness is simulated. The lateral stiffness, provided by the activebending of the lamellae, would be hard to estimate without the explicit modeling of the edge beams, therefore it is neglected. The T-shaped spiral footing (Fig. 12c) is not modeled and has been replaced by a fixed connection on all the faces pointing down. This is also a simplification since several nonlinearities, such as unilateral contacts, are there located. All these connections are activated in phase two, so the patches are internally joined and the bottom faces are attached to the ground. The structure then behave as a whole and redistributes the stress. Being an indoor installation, only the gravity load with characteristic value is applied in phase two. This is also the scenario for which the demonstrator has been optimized.

Stress peaks occur at sharp vertices and kinks, but these are not deemed to reproduce the reality and are simply a consequence of the automatic generation of the model. To provide a qualitative idea of the material utilization, Fig. 15 reports a histogram the equivalent von Mises stress, which shows that the material is employed in a small spectrum of values. However, von Mises stresses are not meaningful for an orthotropic material, therefore the stress is recalculated for each patch accounting for the fibers direction in which 
Integrated computational framework for bending-active structures

Table 1

Orthotropic material properties adopted in the FE analysis of the demonstrator

\begin{tabular}{ccccccccc}
\hline \multicolumn{2}{c}{$\begin{array}{c}\text { Section Properties } \\
\text { Nominal thickness }\end{array}$} & Density & \multicolumn{2}{c}{ Rigidity } \\
$(\mathrm{mm})$ & & \multicolumn{2}{c}{$\boldsymbol{G}_{0}$} & $\boldsymbol{G}_{90}$ & $\boldsymbol{G}_{\text {out }}$ & $E_{0}$ & $E_{90}$ & $E_{\text {out }}$ \\
\hline 15 & 7 & 520 & 248.4 & 213.6 & 600 & 11067.6 & 9932.4 & 600 \\
\hline
\end{tabular}

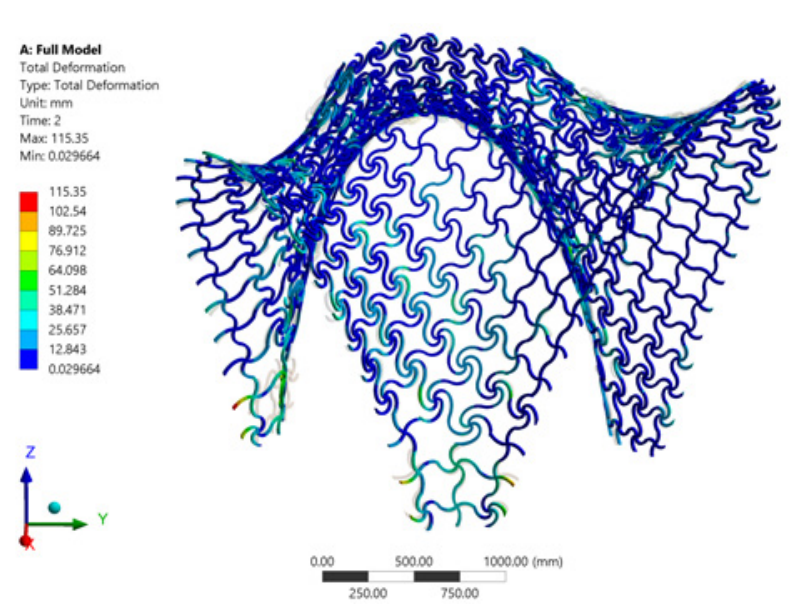

(a)
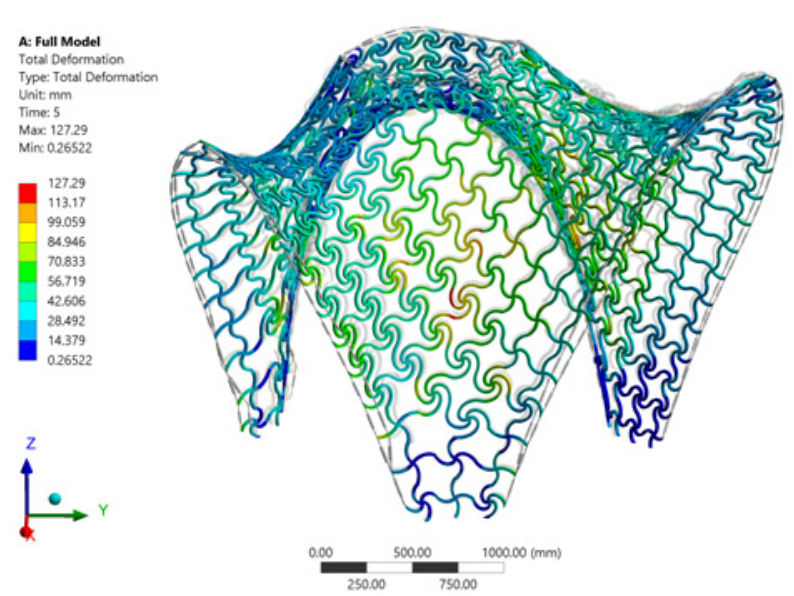

(b)

Figure 14: FE analysis results: (a) displacements in phase 1 and (b) phase 2. The springs are activated after the phase 1.

the strength is tested. Fig. 16 reports these results (along the grain, across the grain and in-plane shear) for an exemplary case of one of the patches attached to the ground bent towards the outer direction ( $\mathrm{Z}$ in the patch local frame), showing one of the most stressed areas of the structure. Due to bending, inner ( $\mathrm{Z}$ positive) and outer ( $\mathrm{Z}$ negative) faces present high, negative or positive respectively, stress values. The material strength has not been experimental investigated, however the obtained stress is within the usual nominal capacity of this kind of material, if peaks due to modeling are neglected. Moreover, as a further confirmation of this result, no visible failures appeared during the assembly and in the as-built structure.

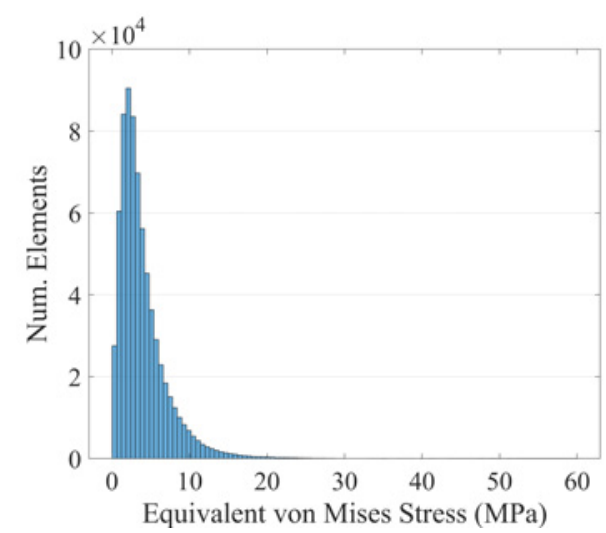

Figure 15: FE analysis results: histogram of phase 2 equivalent von Mises stress.

\subsection{Fabrication and assembly}

The interface between optimized design and fabrication is managed through a Grasshopper workflow. The process starts with a set of planar mesh, which is the outcome of the optimization and parametrization (flattening) step. Each mesh boundary is converted into a NURBS, and the free extremities of the spiral arms are replaced with the three different connector types: a) inner joint to mutually connect the patches; b) an open boundary connector to enable the installation of the edge beams; c) a footing to connect the spirals to the ground beams (Fig. 17). Additionally, the workflow marks each spiral with a numeric label to be milled at its center.

At the fabrication level, the setting of milling precision, tool depth, and tolerances have been considered to avoid surface damage. These variables have a significant role since they affect both the node's structural capacity and its interlocking, especially for the inner connectors. These cutting lines are set to be processed with a $3 \mathrm{~mm}$ diameter tool. The calibration of tolerances between the extremities of the connectors is set to $0.12 \mathrm{~mm}$, which has been deduced experimentally among a set of mock-ups. This choice is a compromise between the necessity to restore the stiffness of a continuous element, which would have required the tightest tolerance possible, and the necessity to be easily installed, which conversely is facilitated by a large tolerance. All the other cutting lines are performed with a $6 \mathrm{~mm}$ diameter tool. The patches and the floor tiles are then nested automatically to minimize the waste of material and sent out for CNC pro- 


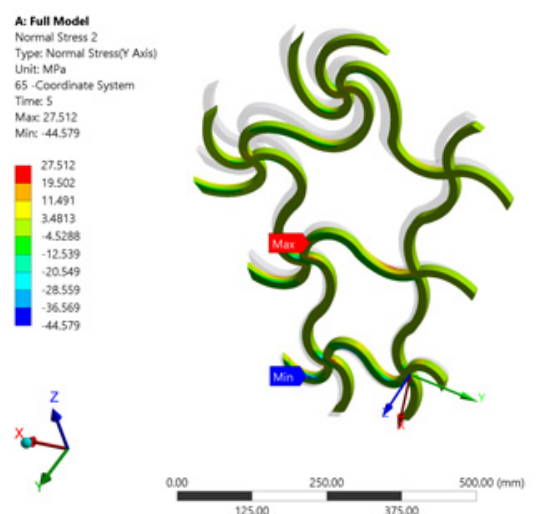

(a)
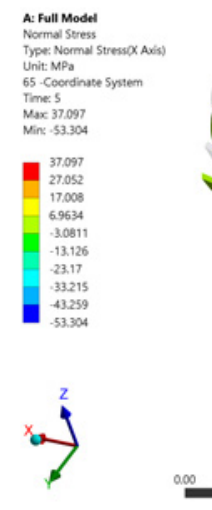

(b)

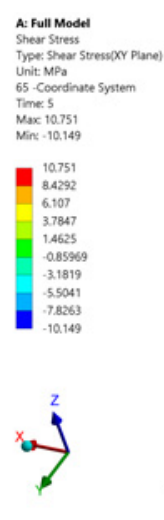

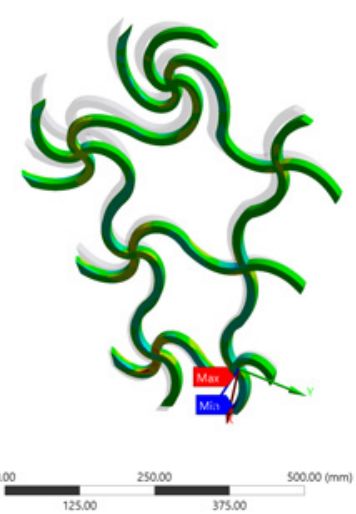

(c)

Figure 16: FE analysis results on panel 65 in phase two (bottom faces are attached to ground): (a) normal stress along the grain; (b) normal stress across the grain; (c) in-plane shear stress. The grain direction is identified as $\mathrm{Y}$ in the patch local frame.

duction. A CNC machine with $3.00 \times 1.60$ meters bed size has been used. Eventually, the only manual post-processing work was to sand all the cut surfaces to remove the wood shavings (Fig. 18).
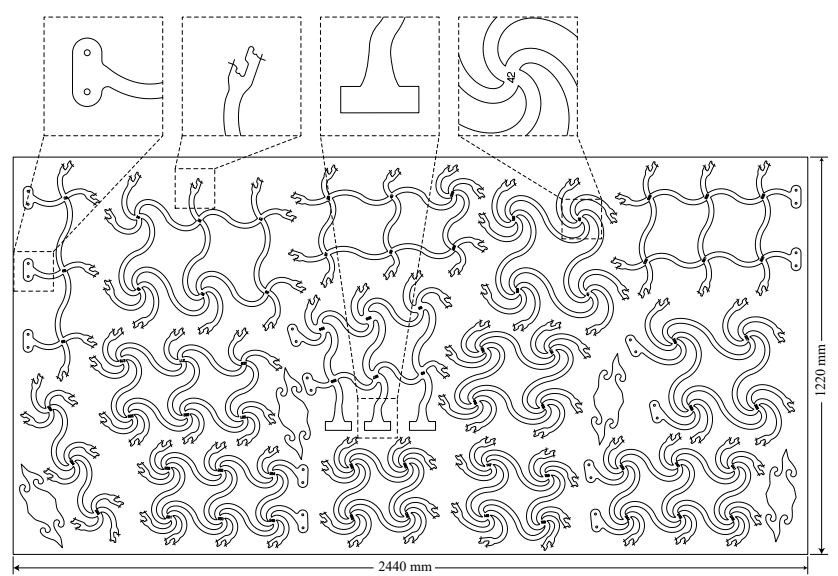

Figure 17: Flat 2D sheet to be cut with highlighted the different connector types (open boundary connector, inner connector and footing, respectively) and the labeling system.

Table 2 includes a summary of the pieces, material, and quantities needed for the assembly of the demonstrator. The assembly phase has been assisted by a 3D model showing the patches and the label of each quad, which corresponds to the milled label on each spiral. Thus, its position could have been uniquely recognized from its adjacencies.

Due to time constraints, a staged analysis of the prototype assembly process has not been performed. Conversely, since the phase one of the structural analysis evidenced no failures, the panels would remain within the material strength range as long as every single patch stays close enough to its target position (deformation state) during the entire assembly procedure.

Therefore, the demonstrator has been assembled starting from the larger saddle, sequentially bending and fastening each
FlexMaps panel. Once this area has been completed, it was secured and stiffened by edge beams. The assembly work moved later on the two other saddles, which have been progressively formed and later stiffened by the edge beams (Fig. 19). At this point, the shape has become stiff enough to be locked onto the ground beams, while the paving and the first layer of the ground beams have been easily assembled as a large puzzle of interlocking tiles.

The entire prototype (Fig. 20) has been designed, optimized, and engineered in about four weeks. However, it has to be considered that some design aspects are not integrated into the main automated pipeline. The fabrication only has taken one week; the assembly employed on average four people for about 18 hours. All the assembly operations have been manually performed and not less than 3-4 people are required in order to bend and connect a couple of patches simultaneously while securing the connectors by bolts. During these operations, 'low-tech' scaffolding, like tables and chairs, have been employed. This was allowed as the shape accuracy relied entirely on the properties of the spirals, the precision of the connections, and the guiding provided by the ground and edge beams.

\subsection{Verification and discussion}

The development of a full scale prototype in a constrained time and space represented a real test for the proposed methodology from both a design and a construction point of view. The computational model has been informed by the experimental tests on the arch specimen, and during this moment the team has familiarized with the basic assembly operations and has gained confidence on how these procedures may affect the final shape of the built structure, as well as any initial imperfection or supports misalignment. However, the arch test has been opportunely designed to avoid such issues and to be easily controlled due to its simple shape and its manageable size.

An interesting verification can be carried out by comparing the shapes of the actual built demonstrator and the de- 

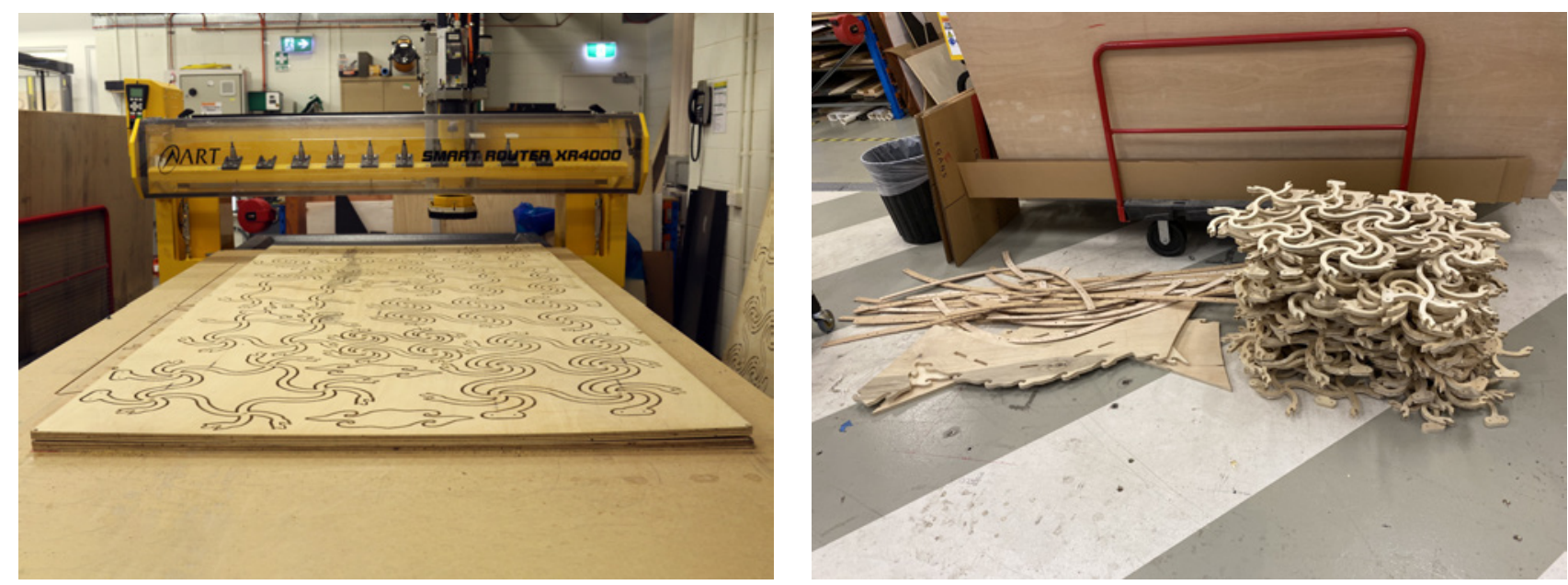

Figure 18: Fabrication of the demonstrator: CNC cutting and components after sanding.

Table 2

Details of the components employed in the assembly of the demonstrator.

\begin{tabular}{|c|c|c|c|c|c|}
\hline & Material & Type & Quantity/Description & Weight & Production time \\
\hline Spiral panels & plywood & 15 mm (width) & 78 bespoke patches & $24 \mathrm{~kg}$ (total) & 5 days \\
\hline Paving tiles & plywood & $15 \mathrm{~mm}$ (width) & $\begin{array}{l}79 \text { tile } A, 43 \text { tile } B, 34 \text { be- } \\
\text { spoke }\end{array}$ & $12.1 \mathrm{~kg}$ (total) & (with previous) \\
\hline Ground beams & plywood & $15 \mathrm{~mm}$ (width) & $\begin{array}{l}3 \text { beams made of three } \\
\text { layers (the top one is seg- } \\
\text { mented) }\end{array}$ & $18.7 \mathrm{~kg}$ (total) & 1 day \\
\hline Edge beams & plywood & $6 \mathrm{~mm}$ (width) & & & 1 day \\
\hline Bolts & Stainless steel & M5 & & & - \\
\hline Screws & Stainless steel & M8 & & & - \\
\hline Ballast discs & Iron & - & & $1 \mathrm{~kg}$ (each) & - \\
\hline
\end{tabular}

formed model obtained as outcome of the FE analysis. During the assembly, the demonstrator could not rely on stepby-step shape checks and on a verified minimal-distortion procedure. The latter would have indeed required a dramatic increase of computational time to run combinatorial staged simulations. Thus, the accuracy of the final shape is totally demanded to the capacity of the panels of matching exactly the target shape they are designed for, to the redistribution of internal stress, and not least to the team working ability. The ground beams and edge beams supported this phase by restraining the inner distance between the edge connectors. To acquire the actually built shape a scan session has been performed one week after the installation through a Artec handheld scanner. The comparison between the FE deformed shape and the as-built object is shown in Fig. 21.

The as-built structure suffers from a large misalignment of the ground beams in the range of $10-30 \mathrm{~mm}$. This is not related to the acquisition procedure but to the tiles' fabrication. In that phase the tolerance between the tiles has not been set properly, and the tiles needed to be filed and forced to fit in place while assembled. Therefore, this error propagates to- wards the ground beams, producing a shifting of the fixed supports of the model that is reasonably responsible of most of the difference between the two shapes. Larger discrepancies are located at the top of the structure. Indeed, this imperfect position of the boundary constraints makes the structure softer, so its inner part next to the hole can displace downward, while the external border is held in place by the edge beams.

To support this evidence another FE model has been recomputed displacing the ground supports to the new position detected by the scanner, given the same orthotropic material properties. The resulting shape is compared with the scan data in Fig. 22. Once the macroscopic imperfection introduced by floor tiles has been included in the model, the overall accuracy has been largely improved, and the deformed shape is matched satisfactorily. However, deviations can be observed locally due to the necessary model simplifications. In fact, the FE model is not represented in all the details that the built structure is endowed with and so it did not embed all the features in both geometry and mechanics: i.e. the connectors, the edge beams and the ground beams 

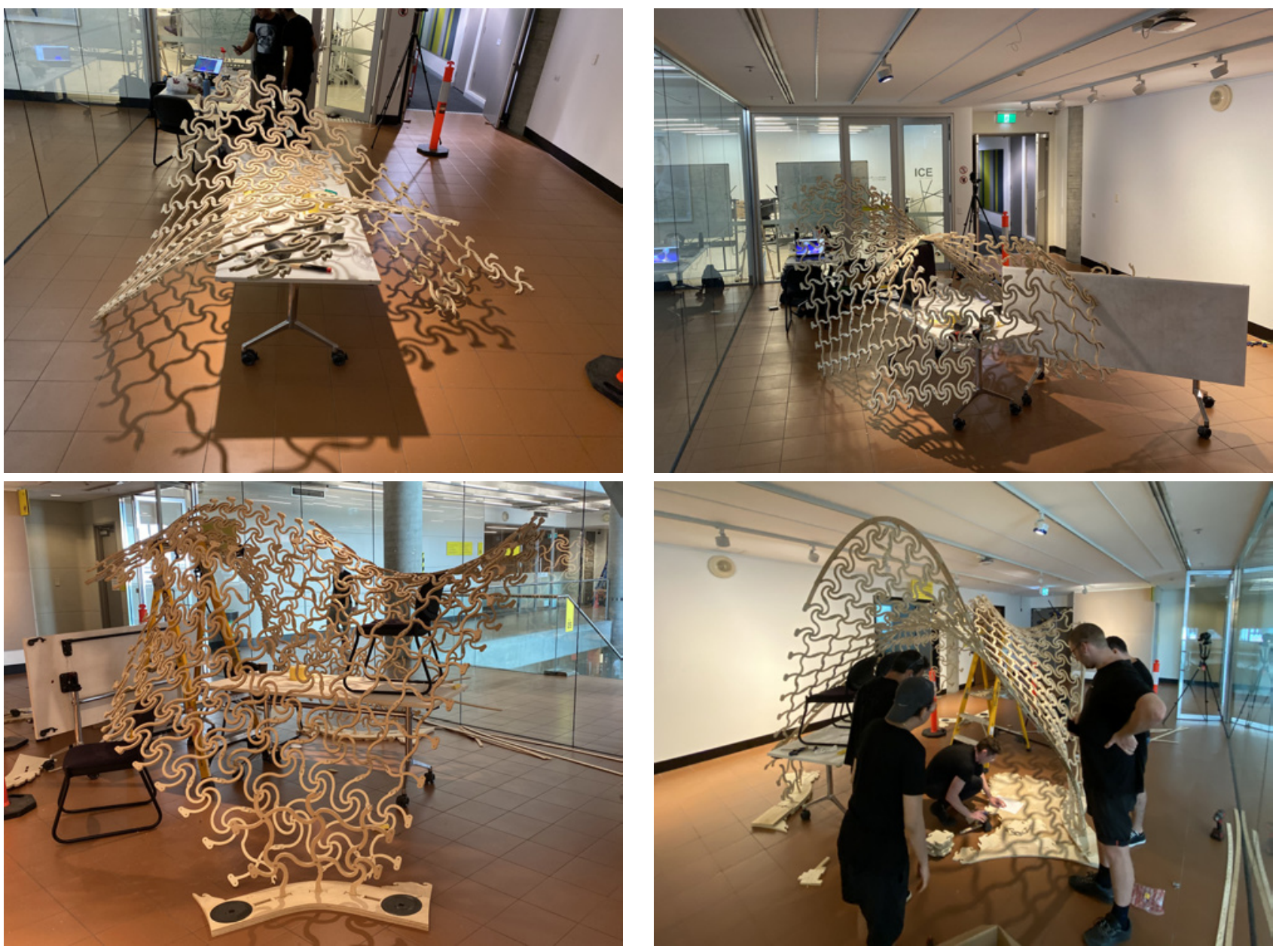

Figure 19: Assembly of the demonstrator.

are simplified, bolts, screws and washers, contact nonlinearities are not included etc. The wood relaxation during time has not been considered. Most importantly, disregarding these factors it appears that the proposed method is effective in terms of matching the target shape. There are no local mismatches that are attributable to the surface quadrangulation or mesostructures form-finding. Therefore, the present pipeline can be considered suitable to produce complex shapes for architectural purposes through bending-active components.

The performance of the demonstrator is also promising from the structural system perspective, even though the choice of layered and anisotropic material such as plywood has brought several issues, as opposed to its strengths the affordable cost and ease of fabrication and handling. From the material stiffness point of view, the selection of wood is in contrast with carved unconventional geometric patterns for two reasons. First, the direction along the grain is stronger than the other across; the result is that the stiffness locally depends on the grain orientation. Second, weak points are also produced if the grain runs across a narrow width, namely the so-called 'short grain' effect; the cuts break the continuity of fibers so the material could not attain its nominal strength. Hav- ing multiple plies and grain in different directions, plywood broadly mitigates these effects (i.e. the Young's modulus differs by about the $10 \%$ along the two directions). This is the reason why plywood has been considered compatible with this specific application and with an optimization routine that is designed for isotropic material behavior. An additional weak point is represented by the edges status. In particular a careful attention has been paid in avoiding local cracks since they can be starting points for tensile failures. This has been achieved by selecting appropriate milling tools and by tuning their velocity. Due to the layered nature of the material, other critical parts are the interfaces of the connectors where the risk of delamination during the assembly is very high.

As main result, the present method has demonstrated feasibility from a technical and a fabrication viewpoint, and good capacity of matching a target shape under certain loads (i.e. gravity) requiring limited assistance during the assembly and few devices to keep the structure in place, such as edge lamellae and ground beams. However, the scaling of this system towards larger spans has to currently deal with a limitation that concerns the behavior of the structure under new loading scenarios. Once the self-shaping process is 

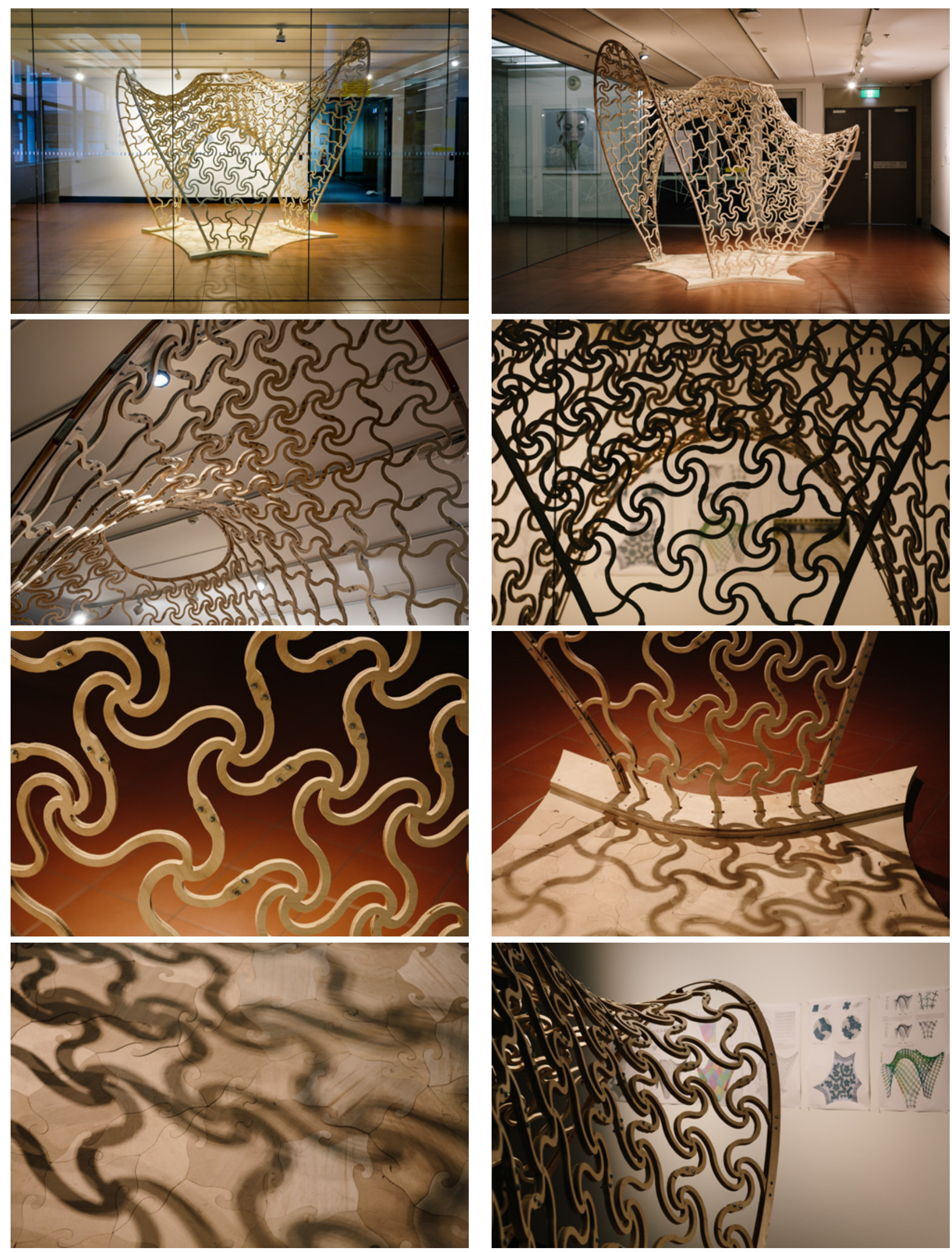

Figure 20: Photos of the completed structure and closeup of the main details of the system. 

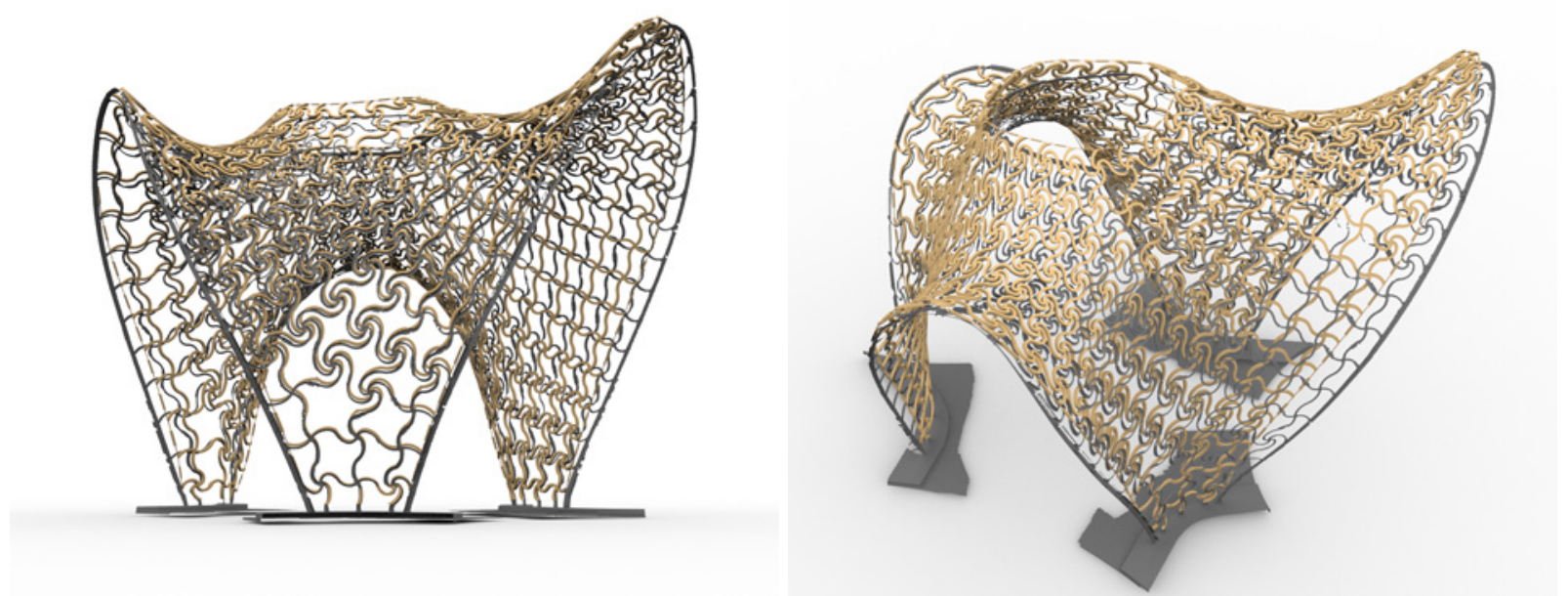

Figure 21: Shape comparison between the theoretical deformed shape (colored) as outcome of the FE analysis and the real scan data (gray).
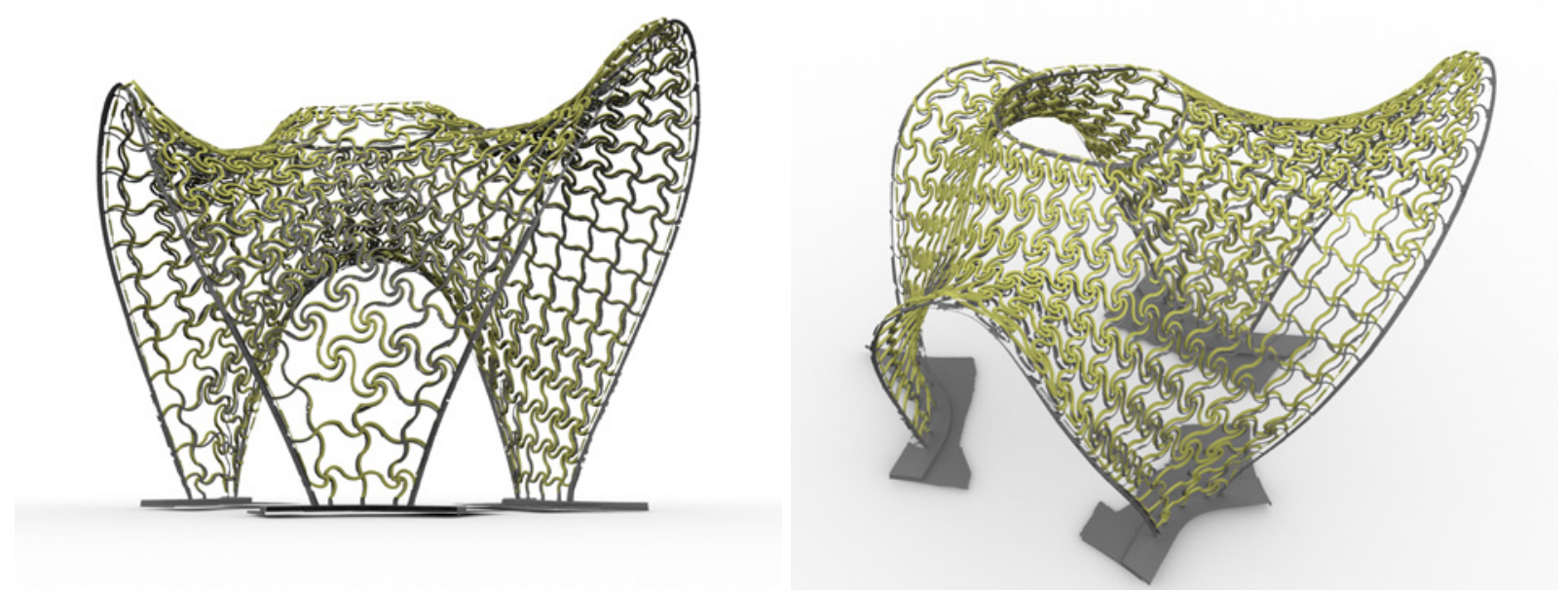

Figure 22: Shape comparison between the theoretical deformed shape (colored) as outcome of the FE analysis and the real scan data (gray). The FE model has been updated considering the new position of the ground faces, as surveyed through the scan session.

concluded, the structure has to develop membrane actions as a lattice shell and to avoid any further bending. The strategies of triggering the membrane behavior due to new loading and its evaluation require future studies.

\section{Conclusions}

This paper introduces a proper architectural pipeline to design and fabricate actively-bent complex structures exhibiting double curvature through cost-effective and time-saving technology. This pipeline modifies and extends the FlexMaps technique, initially tailored for small-scale objects. These structures are provided with custom spiraling mesostructured panels, so that a differentiated local stiffness is achieved by controlling the spiral parameters.

The developed tool allows the designer to reproduce a given target geometry by controlling the structure deformation behavior. This is performed by varying the mesostructure's morphology and the physical behavior of the individual spirals. The framework enables a new way to fabricate bendingactive structures with differentiated material behaviors.

This method can be considered an integral approach to design bending-active structures [25] as it introduces shape and material control in the design phase, as well as filesto-factory pipeline for subtractive fabrication. To demonstrate the method effectiveness and validate the proposed technique a free form structure has been fabricated out of 15$\mathrm{mm}$ thick milled plywood panels. This structure shows the enormous potential of this approach in architecture as it can pave the way for larger structures, providing excellent shape expressiveness while employing common building material.

\section{Acknowledgments}

The authors would gratefully thank Dane Voorderhake, Lucy Wang, Nathan Gonsalves, Ella Williams and the staff 
of the Advanced Fabrication Lab (UTS Faculty of the Architecture), Stefano Nuvoli for the support during the assembly, and Hamish McIntosh and Francesca Giuliani for the demonstrator photos.

\section{Funding}

This research was partially developed during the Short Term Mobility (STM) of Francesco Laccone, hosted by Nico Pietroni at the UTS. The STM scholarship was funded by the Italian National Research Council (CNR).

\section{References}

[1] L. Malomo, J. Pérez, E. Iarussi, N. Pietroni, E. Miguel, P. Cignoni, B. Bickel, FlexMaps: computational design of flat flexible shells for shaping 3D objects, ACM Transactions on Graphics (2018) 241. doi:10.1145/3272127.3275076.

[2] F. Barbosa, J. Woetzel, J. Mischke, Reinventing Construction: A Route of Higher Productivity, Technical Report, McKinsey Global Institute, 2017.

[3] U. Nations, World urbanization prospects: The 2014 revision, highlights. department of economic and social affairs, Population Division, United Nations 32 (2015).

[4] H. Pottmann, Architectural geometry, volume 10, Bentley Institute Press, 2007.

[5] S. Adriaenssens, P. Block, D. Veenendaal, C. Williams, Shell structures for architecture: form finding and optimization, Routledge, 2014.

[6] M. Eigensatz, M. Kilian, A. Schiftner, N. J. Mitra, H. Pottmann, M. Pauly, Paneling Architectural Freeform Surfaces, ACM Transactions on Graphics 29 (2010) 45:1-45:10. doi:10.1145/1778765. 1778782.

[7] Y. Liu, W. Xu, J. Wang, L. Zhu, B. Guo, F. Chen, G. Wang, General Planar Quadrilateral Mesh Design Using Conjugate Direction Field, ACM Transactions on Graphics 30 (2011) 1-10. URL: https://doi. org/10.1145/2070781.2024174. doi:10.1145/2070781.2024174.

[8] M. Deuss, D. Panozzo, E. Whiting, Y. Liu, P. Block, O. SorkineHornung, M. Pauly, Assembling self-supporting structures, ACM Transactions on Graphics 33 (2014) 214:1-214:10. doi:10.1145/ 2661229. 2661266.

[9] M. Skouras, S. Coros, E. Grinspun, B. Thomaszewski, Interactive surface design with interlocking elements, ACM Transactions on Graphics 34 (2015) 224:1-224:7. doi:10.1145/2816795.2818128.

[10] Z. Wang, P. Song, F. Isvoranu, M. Pauly, Design and Structural Optimization of Topological Interlocking Assemblies, ACM Transactions on Graphics 38 (2019). doi:10.1145/3355089. 3356489.

[11] J. N. Richardson, S. Adriaenssens, R. Filomeno Coelho, P. Bouillard, Coupled form-finding and grid optimization approach for single layer grid shells, Engineering Structures 52 (2013) 230-239. URL: https: //www. sciencedirect.com/science/article/pii/s0141029613000813. doi:10.1016/j. engstruct. 2013.02.017.

[12] N. Pietroni, D. Tonelli, E. Puppo, M. Froli, R. Scopigno, P. Cignoni, Statics Aware Grid Shells, Computer Graphics Forum 34 (2015) 627-641. URL: https://onlinelibrary. wiley.com/doi/abs/10.1111/cgf.12590. doi:10.1111/cgf.12590. arXiv:https://onlinelibrary.wiley.com/doi/pdf/10.1111/cgf.12590.

[13] C. Jiang, C. Tang, H.-P. Seidel, P. Wonka, Design and Volume Optimization of Space Structures, ACM Transactions on Graphics 36 (2017) 159:1-159:14. doi:10.1145/3072959.3073619.

[14] N. Pietroni, M. Tarini, A. Vaxman, D. Panozzo, P. Cignoni, Positionbased Tensegrity Design, ACM Transactions on Graphics 36 (2017) 172:1-172:14. doi:10.1145/3130800.3130809.

[15] M. Kilian, D. Pellis, J. Wallner, H. Pottmann, Material-Minimizing Forms and Structures, ACM Transactions on Graphics 36 (2017). doi: $10.1145 / 3130800.3130827$.
[16] F. Gil-Ureta, N. Pietroni, D. Zorin, Reinforcement of general shell structures, ACM Transactions on Graphics 39 (2020). URL: https: //doi.org/10.1145/3375677. doi:10.1145/3375677.

[17] F. Laccone, L. Malomo, M. Froli, P. Cignoni, N. Pietroni, Automatic design of cable-tensioned glass shells, Computer Graphics Forum 39 (2020) 260-273. URL: https://onlinelibrary.wiley.com/doi/abs/10. 1111/cgf. 13801. doi:10.1111/cgf. 13801.

[18] H. Pottmann, Architectural geometry and fabrication-aware design, Nexus Network Journal 15 (2013) 195-208. doi:10.1007/ s00004-013-0149-5.

[19] Y. Liu, H. Pottmann, J. Wallner, Y.-L. Yang, W. Wang, Geometric Modeling with Conical Meshes and Developable Surfaces, ACM Transactions on Graphics 25 (2006) 681-689. doi:10.1145/1141911. 1141941.

[20] K. Gavriil, A. Schiftner, H. Pottmann, Optimizing B-spline surfaces for developability and paneling architectural freeform surfaces, Computer-Aided Design 111 (2019) 29 - 43. doi:10.1016/j.cad. 2019. 01.006.

[21] C. Jiang, C. Wang, F. Rist, J. Wallner, H. Pottmann, Quad-Mesh Based Isometric Mappings and Developable Surfaces, ACM Transactions on Graphics (2020). URL: http: //hdl . handle. net/10754/663008. doi: $10.1145 / 3386569.3392430$.

[22] J. Lienhard, P. Eversmann, New hybrids - from textile logics towards tailored material behaviour, Architectural Engineering and Design Management 0 (2020) 1-6. doi:10.1080/17452007.2020.1744421.

[23] J. Panetta, Q. Zhou, L. Malomo, N. Pietroni, P. Cignoni, D. Zorin, Elastic Textures for Additive Fabrication, ACM Transactions on Graphics 34 (2015). doi:10.1145/2766937.

[24] C. Schumacher, B. Bickel, J. Rys, S. Marschner, C. Daraio, M. H. Gross, Microstructures to control elasticity in 3d printing, ACM Transactions on Graphics 34 (2015) 136:1-136:13. doi:10.1145/ 2766926.

[25] J. Lienhard, H. Alpermann, C. Gengnagel, J. Knippers, Active bending, a review on structures where bending is used as a self-formation process, International Journal of Space Structures 28 (2013) 187-196. doi: $10.1260 / 0266-3511.28 .3-4.187$.

[26] M. Fleischmann, A. Menges, ICD/ITKE Research Pavilion: A Case Study of Multi-disciplinary Collaborative Computational Design, in: C. Gengnagel, A. Kilian, N. Palz, F. Scheurer (Eds.), Computational Design Modelling, Springer Berlin Heidelberg, Berlin, Heidelberg, 2012, pp. 239-248.

[27] K. Crolla, Bending Bamboo Rules: Beyond Century-Old Typologies, Journal of Architectural Education 72 (2018) 135-145. doi:10.1080/ 10464883.2018.1410669.

[28] C. Gengnagel, E. L. Hernández, R. Bäumer, Natural-fibre-reinforced plastics in actively bent structures, Proceedings of the Institution of Civil Engineers-Construction Materials 166 (2013) 365-377. doi:10. 1680/coma.12.00026.

[29] J. Lienhard, R. La Magna, J. Knippers, Form-finding bending-active structures with temporary ultra-elastic contraction elements, WIT Transactions on The Built Environment 136 (2014) 107. doi:10.2495/ MAR140091.

[30] P. Cuvilliers, J. R. Yang, L. Coar, C. Mueller, A comparison of two algorithms for the simulation of bending-active structures, International Journal of Space Structures 33 (2018) 73-85. doi:10.1177/ 0266351118779979.

[31] M. C. Phocas, K. Alexandrou, S. Athini, Design and analysis of an adaptive hybrid structure of linearly interconnected scissor-like and cable bending-active components, Engineering Structures 192 (2019) 156 - 165. URL: http://www. sciencedirect.com/science/article/ pii/s0141029618332796. doi:10.1016/j. engstruct.2019.04.102.

[32] P. Nicholas, M. Tamke, Computational strategies for the architectural design of bending active structures, International Journal of Space Structures 28 (2013) 215-228. doi:10.1260/0266-3511.28.3-4.215.

[33] J. Lienhard, Bending-active structures: form-finding strategies using elastic deformation in static and kinetic systems and the structural potentials therein, 36, Institut für Tragkonstruktionen und Konstruktives Entwerfen (ITKE), Universität Stuttgart, 2014. URL: https: 
//elib.uni-stuttgart.de/handle/11682/124. doi:10.18419/opus-107.

[34] J. Lienhard, J. Knippers, Bending-active textile hybrids, Journal of the International Association for Shell and Spatial Structures 56 (2015) $37-48$.

[35] D. Sonntag, S. Bechert, J. Knippers, Biomimetic timber shells made of bending-active segments, International Journal of Space Structures 32 (2017) 149-159. URL: https://journals. sagepub.com/doi/ 10.1177/0266351117746266. doi:10.1177/0266351117746266.

[36] M. Tamke, Y. S. Baranovskaya, F. Monteiro, J. Lienhard, R. L. Magna, M. R. Thomsen, Computational knit - design and fabrication systems for textile structures with customised and graded CNC knitted fabrics, Architectural Engineering and Design Management 0 (2020) 1-21. URL: https:// doi.org/10.1080/17452007.2020.1747386. doi:10.1080/17452007.2020. 1747386. arXiv:https://doi .org/10.1080/17452007.2020.1747386.

[37] R. La Magna, Bending-active plates: strategies for the induction of curvature through the means of elastic bending of plate-based structures, 43, Institut für Tragkonstruktionen und Konstruktives Entwerfen (ITKE), Universität Stuttgart, 2017. URL: https://elib. uni-stuttgart.de/handle/11682/9406. doi:10.18419/opus-9389.

[38] J. Panetta, M. Konaković-Luković, F. Isvoranu, E. Bouleau, M. Pauly, X-Shells: A New Class of Deployable Beam Structures, ACM Transactions on Graphics 38 (2019). doi:10.1145/3306346.3323040.

[39] F. Laccone, L. Malomo, J. Pérez, N. Pietroni, F. Ponchio, B. Bickel, P. Cignoni, FlexMaps Pavilion: a twisted arc made of mesostructured flat flexible panels, in: Proceedings of IASS Annual Symposia, volume 2019, International Association for Shell and Spatial Structures (IASS), 2019, pp. 1-7.

[40] F. Laccone, L. Malomo, J. Pérez, N. Pietroni, F. Ponchio, B. Bickel, P. Cignoni, A bending-active twisted-arch plywood structure: computational design and fabrication of the FlexMaps Pavilion, SN Applied Sciences 2 (2020). doi:10.1007/s42452-020-03305-w.

[41] N. Pietroni, E. Puppo, G. Marcias, R. Scopigno, P. Cignoni, Tracing field-coherent quad layouts, Computer Graphics Forum 35 (2016) 485-496. URL: https://onlinelibrary. wiley.com/doi/abs/10.1111/cgf.13045. doi:10.1111/cgf.13045. arXiv:https://onlinelibrary. wiley.com/doi/pdf/10.1111/cgf. 13045.

[42] M. S. Floater, K. Hormann, Surface Parameterization: a Tutorial and Survey, in: N. A. Dodgson, M. S. Floater, M. A. Sabin (Eds.), Advances in Multiresolution for Geometric Modelling, Springer, 2005, pp. 157-186.

[43] ANSYS, ANSYS®. Academic Research Mechanical Release 18.0., 2018.

[44] R. McNeel, Associates, Grasshopper generative modeling for Rhino, Computer software, http://www. grasshopper3d. com (2018).

[45] N. Kotelnikova-Weiler, C. Douthe, E. L. Hernandez, O. Baverel, C. Gengnagel, J.-F. Caron, Materials for actively-bent structures, International Journal of Space Structures 28 (2013) 229-240. doi:10. 1260/0266-3511.28.3-4.229. 